\title{
Neutrino quasielastic scattering on nuclear targets
}

\section{Parametrizing transverse enhancement (meson exchange currents)}

\author{
A. Bodek ${ }^{1, a}$, H.S. Budd ${ }^{1}$, M.E. Christy ${ }^{2}$ \\ ${ }^{1}$ Department of Physics and Astronomy, University of Rochester, Rochester, NY 14627-0171, USA \\ ${ }^{2}$ Hampton University, Hampton, VA 23668, USA
}

Received: 3 July 2011 / Revised: 21 July 2011 / Published online: 2 September 2011

(C) The Author(s) 2011. This article is published with open access at Springerlink.com

\begin{abstract}
We present a parametrization of the observed enhancement in the transverse electron quasielastic $(\mathrm{QE})$ response function for nucleons bound in carbon as a function of the square of the four momentum transfer $\left(Q^{2}\right)$ in terms of a correction to the magnetic form factors of bound nucleons. The parametrization should also be applicable to the transverse cross section in neutrino scattering. If the transverse enhancement originates from meson exchange currents (MEC), then it is theoretically expected that any enhancement in the longitudinal or axial contributions is small. We present the predictions of the "Transverse Enhancement" model (which is based on electron scattering data only) for the $v_{\mu}, \bar{v}_{\mu}$ differential and total QE cross sections for nucleons bound in carbon. The $Q^{2}$ dependence of the transverse enhancement is observed to resolve much of the long standing discrepancy in the QE total cross sections and differential distributions between low energy and high energy neutrino experiments on nuclear targets.
\end{abstract}

\section{Introduction}

A reliable description of the neutrino $\left(v_{\mu}\right)$ and antineutrino $\left(\bar{v}_{\mu}\right)$ quasielastic $(\mathrm{QE})$ and inelastic scattering processes (particularly on nuclear targets) is essential for precision studies of $v_{\mu}, \bar{v}_{\mu}$ oscillation [1-6] parameters such as mass splitting and mixing angles. In addition to modeling the $v_{\mu}, \bar{v}_{\mu}$ cross sections [7], a reliable model of the hadronic final states is needed because the hadronic energy response of $v_{\mu}$ detectors is not the same for protons, neutrons, pions, photons, and nuclear fragments. Prescriptions which can be readily incorporated into existing $v_{\mu}$ Monte Carlo generators [8-11] are preferable.

\footnotetext{
a e-mail: bodek@pas.rochester.edu
}

Models which assume that QE scattering on nuclear targets can be described in terms of scattering from independent nucleons bound in a nuclear potential (e.g. Fermi gas [12] or spectral functions) do not provide an adequate representation of measured differential and total QE cross sections for low energy $(\approx 1 \mathrm{GeV}) v_{\mu}$ scattering on nucleons bound in carbon [13-16] (MiniBooNE) and oxygen $[17,18]$ (K2K and T2K). The measured QE total cross sections are $20 \%$ larger than the model and the differential distributions in $Q^{2}$ are also inconsistent. The vector and axial form factors that are used in independent nucleon models are the free nucleon form factors extracted from electron and $v_{\mu}, \bar{v}_{\mu}$ scattering data on hydrogen and deuterium [19].

Although there are more sophisticated calculations of quasielastic scattering (e.g. relativistic distorted-wave impulse approximation [20]), it is the simple independent nucleon model that has been implemented in the currently available neutrino cross section Monte Carlos [8-11].

This disagreement between the measured low energy $v_{\mu}$ differential and total QE cross sections on nuclear targets and the predictions from the independent nucleon model has been attributed to an incomplete description of nuclear effects. These additional nuclear effects have been parametrized as an ad-hoc change in the axial form factor mass parameter from the value measured for free nucleons [19] $\left(M_{A}^{\text {free }}=1.014 \pm 0.014 \mathrm{GeV}\right)$ to $M_{A}^{\text {eff }}=1.20 \pm$ $0.12 \mathrm{GeV}(\mathrm{K} 2 \mathrm{~K})$ and $M_{A}^{\text {eff }}=1.23 \pm 0.20 \mathrm{GeV}$ (MiniBooNE).

A recent analysis $[15,16]$ of newly published differential QE cross sections from MiniBooNE (for nucleons bound in carbon) yields larger values of $M_{A}^{\text {eff }}=1.350 \pm 0.066 \mathrm{GeV}$ in the Fermi gas model and $M_{A}^{e f f}=1.343 \pm 0.060 \mathrm{GeV}$ in the spectral function model. In that analysis the free nucleon value $M_{A}^{\text {free }}=1.014 \mathrm{GeV}$ is excluded at the confidence level greater than $5 \sigma$ (standard deviations). 


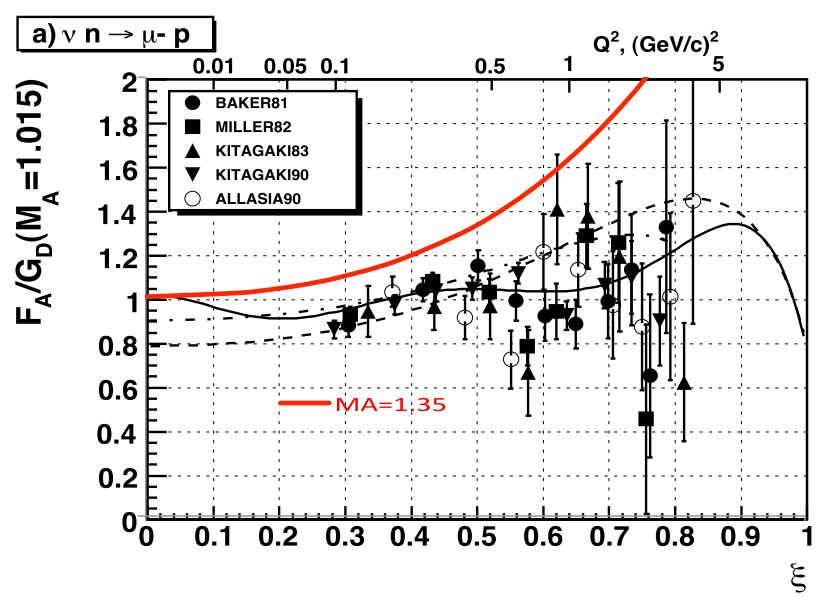

Fig. 1 (a) $F_{A}\left(Q^{2}\right)$ extracted from $v_{\mu}$-deuterium data [19] divided by $G_{D}^{A}\left(Q^{2}\right)$ with $M_{A}=1.015 \mathrm{GeV}$. (b) $F_{A}\left(Q^{2}\right)$ from pion electroproduction (corrected for hadronic effects) divided by $G_{D}^{A}\left(Q^{2}\right)$ with $M_{A}=1.015 \mathrm{GeV}$. Thin solid line-duality based fit from reference [19]; short-dashed line- $F_{A}\left(Q^{2}\right)_{A 2=V 2}$. Dashed-dot line-

Figure 1 shows the world's data [19] for the nucleon axial form factor $\left(F_{A}\left(Q^{2}\right)\right)$ extracted from QE $v_{\mu}, \bar{v}_{\mu}$ scattering on hydrogen and deuterium. Here, the data for $F_{A}\left(Q^{2}\right)$ are shown as a ratio to a nominal dipole $F_{A}\left(Q^{2}\right)=G_{D}^{A}\left(Q^{2}\right)=$ $\frac{-1.267}{\left(1+Q^{2} / M_{A}^{2}\right)^{2}}$ with $M_{A}=1.015 \mathrm{GeV}$. On the left side we show the values extracted from $v_{\mu}, \bar{v}_{\mu}$ experiments on hydrogen and deuterium and on the right side we show the values extracted from pion electro-production data on hydrogen. The average of the measurements of $M_{A}$ from $v_{\mu}, \bar{v}_{\mu}$ experiments on hydrogen and deuterium of $M_{A}^{v_{\mu}, \bar{v}_{\mu}}=$ $1.016 \pm 0.026 \mathrm{GeV}$ is in agreement with the average value of $M_{A}^{\text {pion }}=1.014 \pm 0.016 \mathrm{GeV}$ extracted from pion electroproduction experiments on hydrogen (after corrections for hadronic effects). The average of the $v_{\mu}, \bar{v}_{\mu}$ and electroproduction values is [19] $M_{A}^{\text {world }-a v}=1.014 \pm 0.014 \mathrm{GeV}$. The thin solid line is a duality based parametrization [19] of possible deviations from the dipole form. The dasheddot line is the prediction of a constituent quark model [25] and the short-dashed line is the expectation for $F_{A}\left(Q^{2}\right)$ if the vector and axial-vector structure functions are equal (eg. $\mathcal{W}_{2}^{\text {Qelastic-vector }}=\mathcal{W}_{2}^{\text {Qelastic-axial }}$ ).

It is clearly observed that a dipole axial form factor with $M_{A}=1.35 \mathrm{GeV}$ (thick solid red line) is inconsistent with the measurements on hydrogen and deuterium.

It has been assumed that an "effective" axial mass provides an adequate description of the missing nuclear corrections. However, a large increase in the axial form factor of bound nucleons is contrary to theoretical expectations that $M_{A}$ in nuclear targets should be smaller $[26,27]$ than (or the same [28]) as in deuterium.

Additionally, the low energy neutrino data appear to be in disagreement with higher energy neutrino experiments on

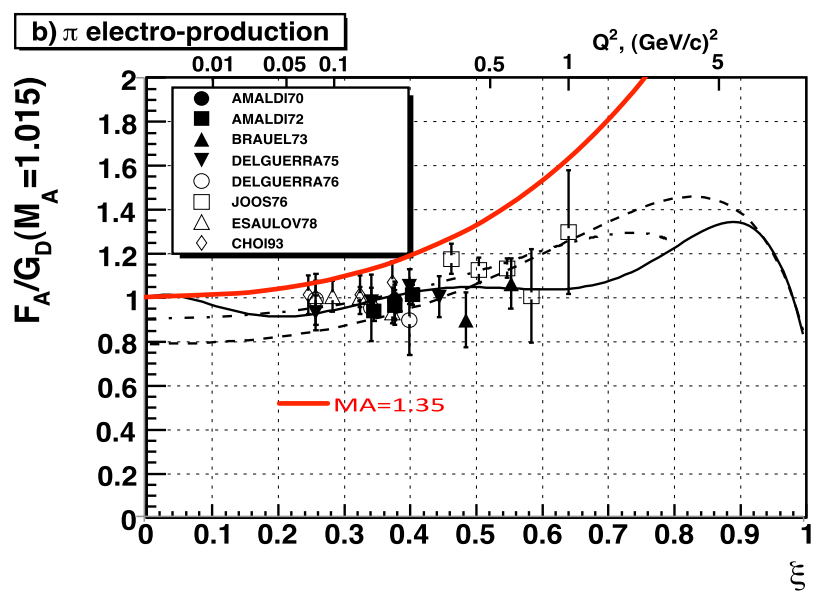

constituent quark model; thick solid red line $F_{A}\left(Q^{2}\right)=G_{D}^{A}\left(Q^{2}\right)=$ $\frac{-1.267}{\left(1+Q^{2} / M_{A}^{2}\right)^{2}}$ with $M_{A}=1.35 \mathrm{GeV}$. The horizontal scale on top is $Q^{2}$. The horizontal scale on the bottom is the target mass scaling variable $\xi$ for elastic scattering $(x=1)$. Here $\xi=\frac{2}{(1+\sqrt{1+1 / \tau})}, \tau=Q^{2} / 4 M^{2}$, and $M$ is the average nucleon mass

nuclear targets. At high neutrino energies, the total and differential QE cross sections on nuclear targets are consistent with models which assume that the scattering is on independent nucleons with free nucleon form factors. For example, $M_{A}$ of $0.979 \pm 0.016 \mathrm{GeV}$ has been extracted from a global analysis [29] of the differential distributions and total QE cross sections measured in all high energy $v_{\mu}$ experiments on nuclear targets.

Recent measurements of the differential and total QE cross section for nucleons bound in carbon by the NOMAD [30, 31] collaboration for $v_{\mu}, \bar{v}_{\mu}$ energies above $4 \mathrm{GeV}$ are also consistent with models which assume that the scattering is from independent nucleons with free nucleon form factors. The NOMAD analysis yields a value of $M_{A}(1.05 \pm 0.02 \pm 0.06 \mathrm{GeV})$.

Therefore, the results of the higher energy and low energy $v_{\mu}$ experiments on nuclear targets appear to be inconsistent with each other.

In this communication we investigate the transverse enhancement observed in QE electron scattering experiments on nuclear targets. We obtain a parametrization of the enhancement and investigate its implication for $v_{\mu}, \bar{v}_{\mu}$ scattering. We show that the $Q^{2}$ dependence of the transverse enhancement resolves much of the discrepancy between the low energy and high energy neutrino experiments, in addition to obviating the need for an ad-hoc nuclear modification to $M_{A}$.

\section{Electron-nucleon scattering}

The differential cross section for scattering of an unpolarized charged lepton with an incident energy $E_{0}$, final energy 
$E^{\prime}$ and scattering angle $\theta$ can be written in terms of the structure functions $\mathcal{F}_{1}$ and $\mathcal{F}_{2}$ as:

$$
\begin{aligned}
& \frac{d^{2} \sigma}{d \Omega d E^{\prime}}\left(E_{0}, E^{\prime}, \theta\right) \\
& =\frac{4 \alpha^{2} E^{\prime 2}}{Q^{4}} \cos ^{2}(\theta / 2) \\
& \quad \times\left[\mathcal{F}_{2}\left(x, Q^{2}\right) / v+2 \tan ^{2}(\theta / 2) \mathcal{F}_{1}\left(x, Q^{2}\right) / M\right]
\end{aligned}
$$

where $\alpha$ is the fine structure constant, $M$ is the nucleon mass, $v=E_{0}-E^{\prime}$ is the energy of the virtual photon which mediates the interaction, $Q^{2}=4 E_{0} E^{\prime} \sin ^{2}(\theta / 2)$ is the invariant four-momentum transfer squared, and $x=Q^{2} / 2 M v$ is the Bjorken scaling variable. We define $\mathcal{F}_{2}=\nu \mathcal{W}_{2}, \mathcal{F}_{1}=$ $M \mathcal{W}_{1}$ (and for $v_{\mu}, \bar{v}_{\mu}$ scattering $\mathcal{F}_{3}=\nu \mathcal{W}_{3}$ ).

Alternatively, one could view this scattering process as virtual photon absorption. Unlike the real photon, the virtual photon can have two modes of polarization. In terms of the cross section for the absorption of transverse $\left(\sigma_{T}\right)$ and longitudinal $\left(\sigma_{L}\right)$ virtual photons, the differential cross section can be written as,

$\frac{d^{2} \sigma}{d \Omega d E^{\prime}}=\Gamma\left[\sigma_{T}\left(x, Q^{2}\right)+\epsilon \sigma_{L}\left(x, Q^{2}\right)\right]$

where,

$\Gamma=\frac{\alpha K E^{\prime}}{4 \pi^{2} Q^{2} E_{0}}\left(\frac{2}{1-\epsilon}\right)$

$\epsilon=\left[1+2\left(1+\frac{Q^{2}}{4 M^{2} x^{2}}\right) \tan ^{2} \frac{\theta}{2}\right]^{-1}$

$K=\frac{2 M \nu-Q^{2}}{2 M}$

The quantities $\Gamma$ and $\epsilon$ represent the flux and the degree of longitudinal polarization of the virtual photons respectively, which the quantity $R$ is defined as the ratio $\sigma_{L} / \sigma_{T}$, and is related to the structure functions by

$R\left(x, Q^{2}\right)=\frac{\sigma_{L}}{\sigma_{T}}=\frac{\mathcal{F}_{2}}{2 x \mathcal{F}_{1}}\left(1+\frac{4 M^{2} x^{2}}{Q^{2}}\right)-1=\frac{\mathcal{F}_{L}}{2 x \mathcal{F}_{1}}$

where $\mathcal{F}_{L}$ is called the longitudinal structure function. The structure functions are expressed in terms of $\sigma_{L}$ and $\sigma_{T}$ as follows:

$$
\begin{aligned}
& \mathcal{F}_{1}=\frac{M K}{4 \pi^{2} \alpha} \sigma_{T} \\
& \mathcal{F}_{2}=\frac{v K\left(\sigma_{L}+\sigma_{T}\right)}{4 \pi^{2} \alpha\left(1+\frac{Q^{2}}{4 M^{2} x^{2}}\right)} \\
& \mathcal{F}_{L}\left(x, Q^{2}\right)=\mathcal{F}_{2}\left(1+\frac{4 M^{2} x^{2}}{Q^{2}}\right)-2 x \mathcal{F}_{1}
\end{aligned}
$$

or,

$2 x \mathcal{F}_{1}=\mathcal{F}_{2}\left(1+\frac{4 M^{2} x^{2}}{Q^{2}}\right)-\mathcal{F}_{L}\left(x, Q^{2}\right)$

In addition, $2 x \mathcal{F}_{1}$ is given by

$2 x \mathcal{F}_{1}\left(x, Q^{2}\right)=\mathcal{F}_{2}\left(x, Q^{2}\right) \frac{1+4 M^{2} x^{2} / Q^{2}}{1+R\left(x, Q^{2}\right)}$

or equivalently

$\mathcal{W}_{1}\left(x, Q^{2}\right)=\mathcal{W}_{2}\left(x, Q^{2}\right) \frac{1+v^{2} / Q^{2}}{1+R\left(x, Q^{2}\right)}$

In the case of elastic scattering from free nucleons $(x=$ $\left.Q^{2} / 2 M v=1\right)$ the structure functions are related to the nucleon form factors by the following expressions [32]:

$\mathcal{W}_{1 p}^{\text {elastic }}=\delta\left(v-\frac{Q^{2}}{2 M}\right) \tau\left|G_{M p}\left(Q^{2}\right)\right|^{2}$

$\mathcal{W}_{1 n}^{\text {elastic }}=\delta\left(v-\frac{Q^{2}}{2 M}\right) \tau\left|G_{M n}\left(Q^{2}\right)\right|^{2}$

and

$\mathcal{W}_{2 p}^{\text {elastic }}=\delta\left(v-\frac{Q^{2}}{2 M}\right) \frac{\left[G_{E p}\left(Q^{2}\right)\right]^{2}+\tau\left[G_{M p}\left(Q^{2}\right)\right]^{2}}{1+\tau}$

$\mathcal{W}_{2 n}^{\text {elastic }}=\delta\left(v-\frac{Q^{2}}{2 M}\right) \frac{\left[G_{E n}\left(Q^{2}\right)\right]^{2}+\tau\left[G_{M n}\left(Q^{2}\right)\right]^{2}}{1+\tau}$

$R_{p, n}^{\text {elastic }}\left(x=1, Q^{2}\right)=\frac{\sigma_{L}^{\text {elastic }}}{\sigma_{T}^{\text {elastic }}}=\frac{4 M^{2}}{Q^{2}}\left(\frac{G_{E}^{2}}{G_{M}^{2}}\right)$

Here, $\tau=Q^{2} / 4 M_{p, n}^{2}$, where $M_{p, n}$ are the masses of proton and neutron. Therefore, $G_{M p}$ and $G_{M n}$ contribute to the transverse virtual photo-absorption cross section, and $G_{E p}$ and $G_{E n}$ contribute to the longitudinal cross section.

\section{Nucleon form factors}

The nucleon electromagnetic form factors are best described by the $B B B A 2007_{25}$ duality based parametrization [19]. The deviations from the dipole form factors are parametrized by multiplicatives functions $A_{N}(\xi)$ for each of the proton and neutron form factors $\left(A_{E p}\left(\xi^{p}\right), A_{M p}\left(\xi^{p}\right), A_{E n}\left(\xi^{n}\right)\right.$, and $\left.A_{M n}\left(\xi^{n}\right)\right)$. Here, $A_{N}(\xi)=1$ for pure dipole form factors. The variable $\xi$ is the target mass scaling variable for elastic scattering $(x=1)$, where

$\xi^{p, n}=\frac{2}{\left(1+\sqrt{1+1 / \tau_{p, n}}\right)}$ 

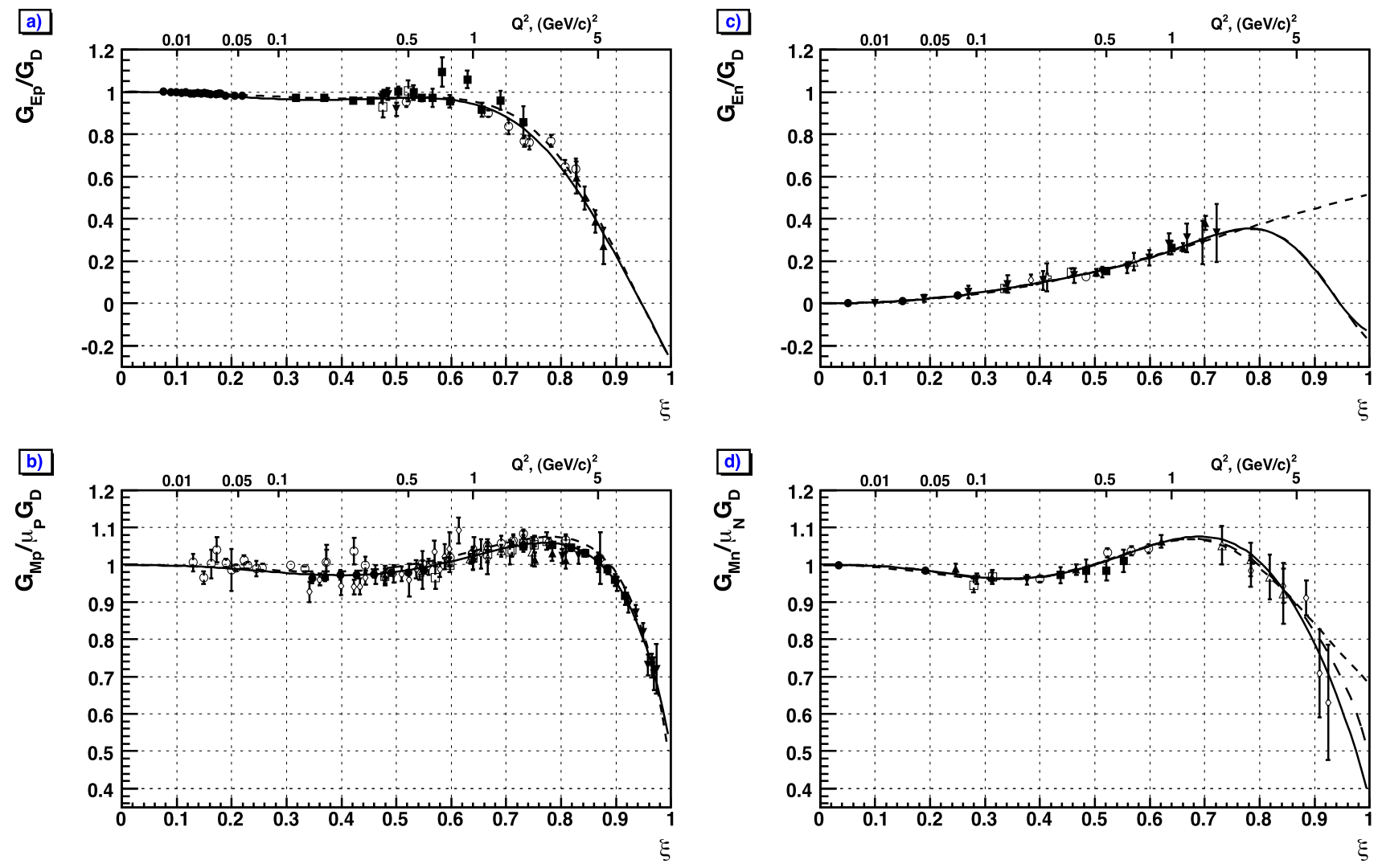

Fig. 2 Ratios of $G_{E p}(\mathbf{a}), G_{M p} / \mu_{p}(\mathbf{b}), G_{E n}(\mathbf{c})$ and $G_{M n} / \mu_{n}$ (d) to $G_{D}^{V}=\frac{1}{\left(1+Q^{2} / M_{V}^{2}\right)^{2}}$ with $M_{V}=0.8426 \mathrm{GeV}$. The short-dashed line in each plot is the old Kelly [21] parameterizations (old Galster [22-24]

and $\tau_{p, n}=Q^{2} / 4 M_{p, n}^{2}$. Here $M_{p, n}$ are the proton $\left(0.9383 \mathrm{GeV} / \mathrm{c}^{2}\right)$ and neutron $\left(0.9396 \mathrm{GeV} / \mathrm{c}^{2}\right)$ masses, respectively.

$$
\begin{aligned}
& G_{D}^{V}\left(Q^{2}\right) \equiv \frac{1}{\left(1+Q^{2} / M_{V}^{2}\right)^{2}} \\
& G_{E p}\left(Q^{2}\right)=A_{E p \text {-dipole }}\left(\xi^{p}\right) \times G_{D}^{V}\left(Q^{2}\right) \\
& G_{E n}\left(Q^{2}\right)=A_{E n}^{25}\left(\xi^{n}\right) \times G_{E p}\left(Q^{2}\right) \times\left(\frac{a \tau_{n}}{1+b \tau_{n}}\right) \\
& G_{M p}\left(Q^{2}\right) / \mu_{p}=A_{M p-\text { dipole }}\left(\xi^{p}\right) \times G_{D}^{V}\left(Q^{2}\right) \\
& G_{M n}\left(Q^{2}\right) / \mu_{n}=A_{M n}^{25}\left(\xi^{n}\right) \times G_{M p}\left(Q^{2}\right) / \mu_{p}
\end{aligned}
$$

Here $\mu_{p}=2.7928, \mu_{n}=-1.913$, and $M_{V}^{2}=0.71 \mathrm{GeV}^{2}$ $\left(M_{V}=0.8426 \mathrm{GeV}\right)$. The parameters for the multiplicative functions $A_{N}(\xi)$ which describes the ratio to dipole are given in reference [19]. The parametrizations are compared to experimental data in Fig. 2.

For the axial form factor we use

$$
F_{A}\left(Q^{2}\right)=G_{D}^{A}\left(Q^{2}\right)=\frac{g_{a}}{\left(1+Q^{2} / M_{A}^{2}\right)^{2}}
$$

for $\left.G_{E n}\right)$. The solid line is the $B B B A 07_{25}$ and the long-dashed line is $B B B A 07_{43}$ parametrizations [19], respectively. The values of $\xi$ and the corresponding values of $Q^{2}$ are shown on the bottom and top axis

where $g_{A}=-1.267$, and $M_{A}=1.014 \pm 0.014 \mathrm{GeV}$ is the axial mass for free nucleons.

The ratio of longitudinal and transverse cross sections for free nucleons is given by:

$$
\begin{aligned}
& R_{p}^{\text {elastic }}=\frac{4 M^{2} / \mu_{p}^{2}}{Q^{2}} \frac{A_{E p \text {-dipole }}^{2}}{A_{M p \text {-dipole }}^{2}}=\frac{0.481}{Q^{2}} \frac{A_{E p \text {-dipole }}^{2}}{A_{M p \text {-dipole }}^{2}} \\
& R_{n}^{\text {elastic }}=\frac{\mu_{p}^{2}}{\mu_{n}^{2}} R_{p}^{\text {elastic }} \frac{\left(A_{E n}^{25}\right)^{2}}{\left(A_{M n}^{25}\right)^{2}}\left(\frac{a \tau_{n}}{1+b \tau_{n}}\right)^{2}
\end{aligned}
$$

In the dipole approximation with $G_{E n}=0$

$$
R_{\text {deuteron }}^{\text {elastic }} \approx \frac{4 M^{2} /\left(\mu_{p}^{2}+\mu_{n}^{2}\right)}{Q^{2}}=\frac{0.328}{Q^{2}}
$$

\section{Quasielastic electron scattering from nuclear targets}

For electron-nucleon and muon-nucleon scattering, scattering from free nucleons (with no pions in the final state) is called elastic scattering, and scattering from nucleons bound in a nuclear target (with no pions in the final state) is called 
QE scattering because the scattering is from quasi-free nucleons.

For charged-current $v_{\mu}$-nucleon and $\bar{v}_{\mu}$-nucleon scattering (with no pions in the final state), the term QE scattering is used to describe scattering from either free or bound nucleons because the neutrino is transformed to a final state muon. For neutrino processes, the term elastic scattering is only used when there is a neutrino in the final state.

Studies of QE electron scattering on nuclear targets indicate that only the longitudinal part of the $\mathrm{QE}$ cross section can be described in terms of a universal response function of independent nucleons bound in a nuclear potential [33] (and free nucleon form factors). In contrast, a significant additional enhancement with respect to the model is observed in the transverse part of the $\mathrm{QE}$ cross section.

The enhancement in the transverse QE cross section has been attributed to meson exchange currents (MEC) in a nucleus [33-38]. Meson exchange currents originate from nucleon-nucleon correlations (predominantly neutron- proton). The final state for the MEC process can include one or two nucleons. If no final state pions are produced, the process is considered as an enhancement of the QE cross section. If one or more final state pions are produced, the process enhances the inelastic cross section.

Within models of meson exchange currents the enhancement is primarily in the transverse part of the QE cross section, while the enhancement in the longitudinal QE cross section is small (in agreement with the electron scattering experimental data). The conserved vector current hypothesis (CVC) implies that the corresponding vector structure function for the QE cross section in $v_{\mu}, \bar{v}_{\mu}$ scattering can be expressed in terms of the structure functions measured in electron scattering on nuclear targets. Therefore, there should also be a transverse enhancement in neutrino scattering.

In addition, for some models of meson exchange currents [36] the enhancement in the axial part of $v_{\mu}, \bar{v}_{\mu} \mathrm{QE}$ cross section on nuclear targets is also small. Therefore, the axial form factor for bound nucleons is expected to be the same as the axial form factor for free nucleons.

\subsection{Measuring the transverse enhancement at low $Q^{2}$}

The longitudinal response scaling functions extracted by Donnely et al. [33] for different momentum scales and different nuclei ( $A=12,40$ and 56) are essentially described by one universal curve [33] which is a function of the nuclear scaling variable $\psi^{\prime}$ only. The function peaks at $\psi^{\prime}=0$ and ranges from $\psi^{\prime}=-1.2$ to $\psi^{\prime}=2$. In contrast, the transverse response scaling function is larger and increases with momentum transfer. The response function of the transverse enhancement excess is shifted to higher $\psi^{\prime}$ and peaks at $\psi^{\prime} \approx 0.2$.

Carlson et al. [36] uses the measured longitudinal and transverse response functions to extract the ratio $\left(\mathcal{R}_{T}\right)$ of the integrated response functions for the transverse and transverse components of the $\mathrm{QE}$ response functions for values of $\psi^{\prime}<0.5$ and $\psi^{\prime}<1.2$.

For nucleons bound in carbon, the ratios for $\psi^{\prime}<0.5$ are $1.2,1.5,1.65$ for values of the 3-momentum transfer $q_{3}$ of $0.3,0.5$, and $0.6 \mathrm{GeV} / \mathrm{c}$, respectively $\left(q_{3}^{2}=Q^{2}+v^{2}\right.$ where $v=Q^{2} / 2 M$ at the $\mathrm{QE}$ peak).

The ratios for $\psi^{\prime}<1.2$ are $1.25,1.6,1.8$ for $q_{3}$ values of $0.3,0.5$, and $0.6 \mathrm{GeV}$, respectively. (These correspond to $Q^{2}$ values of $0.09,0.15$, and 0.33 .) At higher values of $\psi^{\prime}$ the transverse response functions include both $\mathrm{QE}$ scattering and pion production processes (e.g. $\Delta$ production with Fermi motion).

Therefore, we use the measured values of $\mathcal{R}_{T}$ for $\psi^{\prime}<$ 0.5 , where the contribution from pion production process is small, and apply correction to extract the ratio for the entire range of $\psi^{\prime}$, as described below.

The excess transverse response function peaks at $\psi^{\prime} \approx$ 0.2 , while the longitudinal response function peaks at $\psi^{\prime}=0$. A fit of an asymmetric gaussian to the longitudinal response function indicates that the $\mathcal{R}_{T}$ values for the total response functions integrated over all $\psi^{\prime}$ are related to the ratio for $\psi^{\prime}<0.5$ by the following expression:

$\mathcal{R}_{T}\left(\right.$ all $\left.-\psi^{\prime}\right)=1+1.18\left[\mathcal{R}_{T}\left(\psi^{\prime}<0.5\right)-1\right]$

We obtain $\mathcal{R}_{T}\left(\right.$ all $\left.-\psi^{\prime}\right)$ values of $1.24 \pm 0.1,1.59 \pm 0.1$, and $1.77 \pm 0.1$ for $Q^{2}$ values of $0.09,0.15$, and $0.33(\mathrm{GeV} / \mathrm{c})^{2}$, respectively. We use the difference in the measured values of $\mathcal{R}_{T}$ for $\psi^{\prime}<0.5$ and $\psi^{\prime}<1.2$ as an estimate of the systematic error. Since the longitudinal response function is equal to the response function for independent nucleons, the ratio $\mathcal{R}_{T}\left(\right.$ all $\left.-\psi^{\prime}\right)$ is equivalent to the ratio of the integrated transverse response function in a nucleus to the response function for independent nucleons (as a function of $Q^{2}$ ).

The values of $\mathcal{R}_{T}$ extracted from the data of from Carlson et al. are shown as a function of $Q^{2}$ (black points) in Fig. 3.

\subsection{Measuring the transverse enhancement at high $Q^{2}$}

The technique of using the ratio of longitudinal and transverse $\mathrm{QE}$ structure functions to determine the transverse enhancement in the response functions for $\mathrm{QE}$ scattering is less reliable for $Q^{2}>0.5(\mathrm{GeV} / \mathrm{c})^{2}$, because at high values of $Q^{2}$ the longitudinal contribution to the $\mathrm{QE}$ cross section is small (as illustrated in (10)).

Since the transverse cross section dominates at large $Q^{2}$ one can extract the transverse enhancement by comparing the measured $\mathrm{QE}$ cross sections to the predictions of the independent nucleon model directly. However, because there is overlap between pion production processes and $\mathrm{QE}$ 
Transverse Enhancement Carbon 12

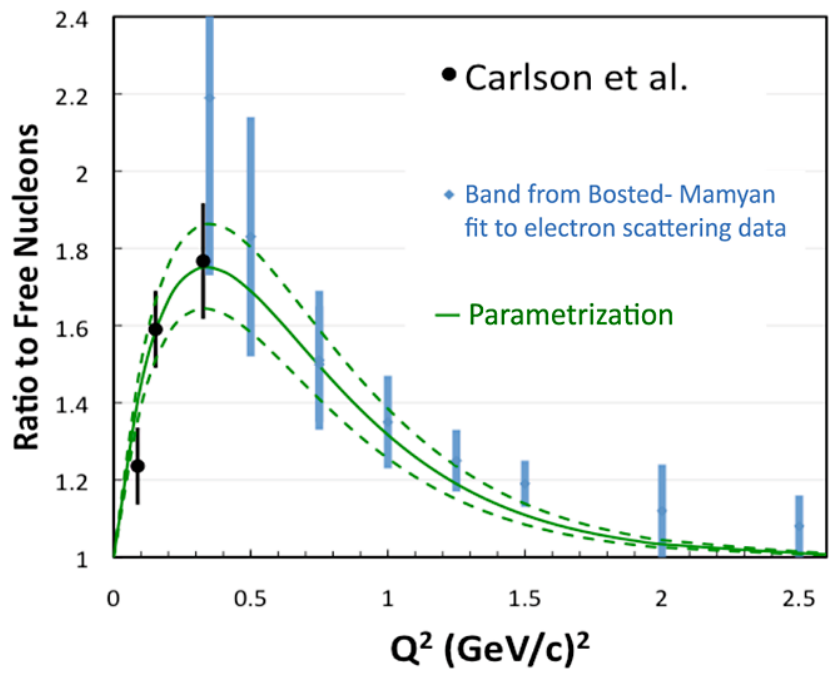

Fig. 3 The transverse enhancement ratio $\left(\mathcal{R}_{T}\right)$ as a function of $Q^{2}$. Here, $\mathcal{R}_{T}$ is ratio of the integrated transverse response function for $\mathrm{QE}$ electron scattering on nucleons bound in carbon divided by the integrated response function for independent nucleons. The black points are extracted from Carlson et al. [36], and the blue bands are extracted from a fit [43] to QE data from the JUPITER [39] experiment (Jlab experiment E04-001). The curve is a fit to the data of the form $\mathcal{R}_{T}=1+A Q^{2} e^{-Q^{2} / B}$. The dashed lines are the upper and lower error bands

scattering, the contribution from pion production processes should be accounted for in the extraction process.

We extract the transverse enhancement at higher values of $Q^{2}$ from a fit to existing electron scattering differential cross sections on nuclei, including preliminary data from the JUPITER collaboration [39] (Jefferson lab experiment E04-001). The fit (developed by P. Bosted and V. Mamyan) provides a description of inclusive electron scattering differential cross sections on a range of nuclei with $A>2$. It is an extension of fits to the free proton [41] and deuteron [42] cross sections. The fit is utilized for calculations of the radiative corrections for the JUPITER analysis [43]. Experiment E04-001 was designed to provide separations of the longitudinal and transverse structure functions from a range of nuclei. These data, therefore, provides a significant constraint on this separation in both the quasi-elastic and resonance regions, which are of critical importance for the current study. A brief description of the fit is given in [43] (where plots of the fit residuals to the data sets utilized are presented).

The inclusive fit is a sum of four components:

- The longitudinal QE contribution calculated for independent nucleons (smeared by Fermi motion in carbon)

- The transverse QE contribution calculated for independent nucleons (smeared by Fermi motion in carbon)

- The contribution of inelastic pion production processes (smeared by Fermi motion in carbon).
- A transverse excess (TE) contribution (determined by the fit)

The QE model used in the Bosted-Mamyan fit is the super-scaling model [40] of Sick, Donnelly, and Maieron.

Figures 4 and 5 show samples of Bosted-Mamyan fits to preliminary electron scattering differential cross sections from JUPITER on a carbon target. Shown are the contributions from the transverse QE (solid pink), longitudinal QE (dashed pink), total QE (solid red), inelastic pion production processes (solid green), and a transverse excess (TE) contribution (solid black line).
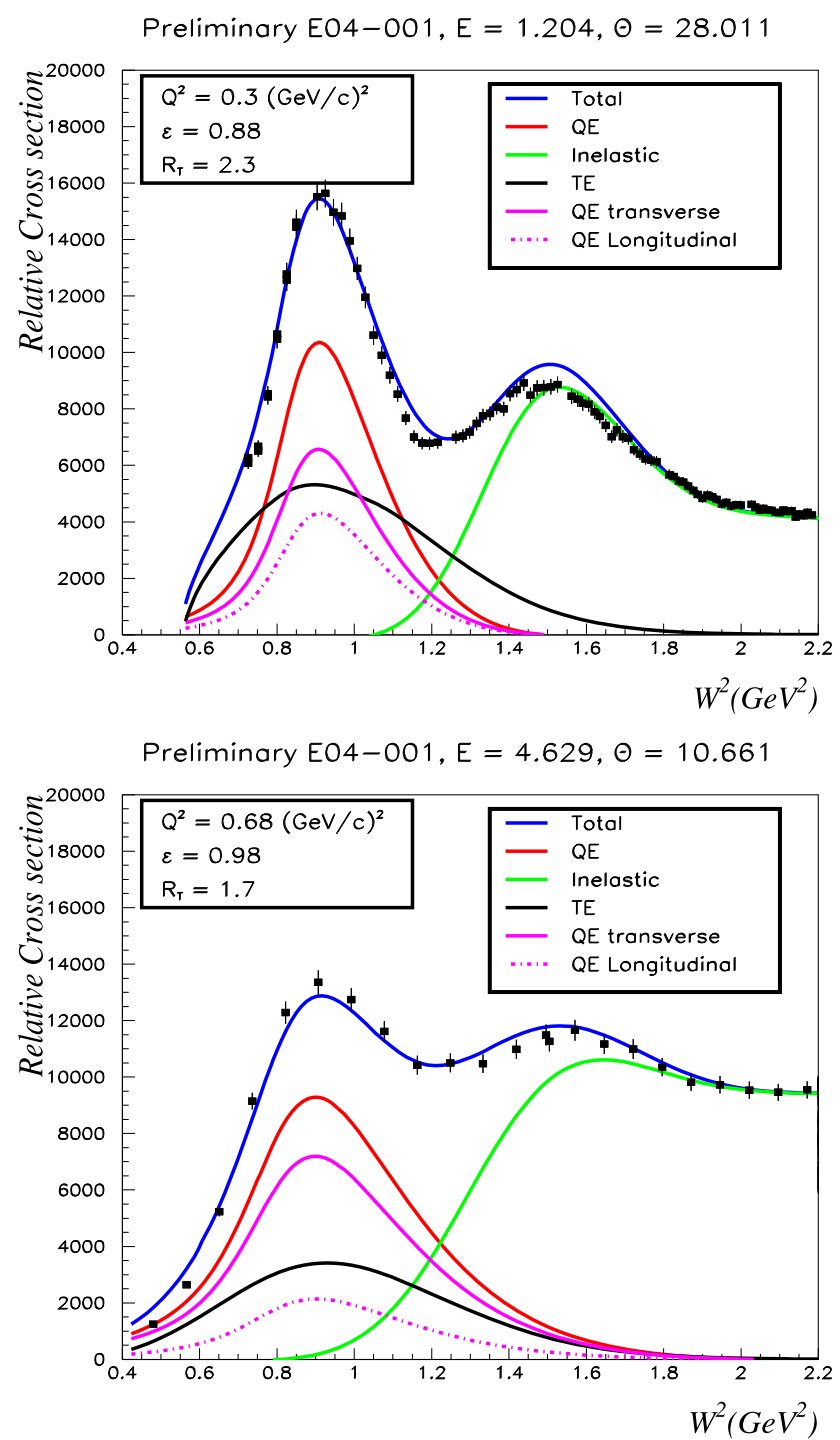

Fig. 4 Samples of fits [43] to preliminary electron scattering data from the JUPITER collaboration [39] (Jefferson Lab experiment E04-001) on a carbon target. Shown are the contributions from the transverse QE (solid pink), longitudinal QE (dashed pink), total QE (solid red), inelastic (pion production) processes (solid green), and a transverse excess (TE) contribution (solid black line). Top: $Q^{2}=0.3 \mathrm{GeV} / \mathrm{c}^{2}$ at the QE peak. Bottom: $Q^{2}=0.68 \mathrm{GeV} / \mathrm{c}^{2}$ at the $\mathrm{QE}$ peak 


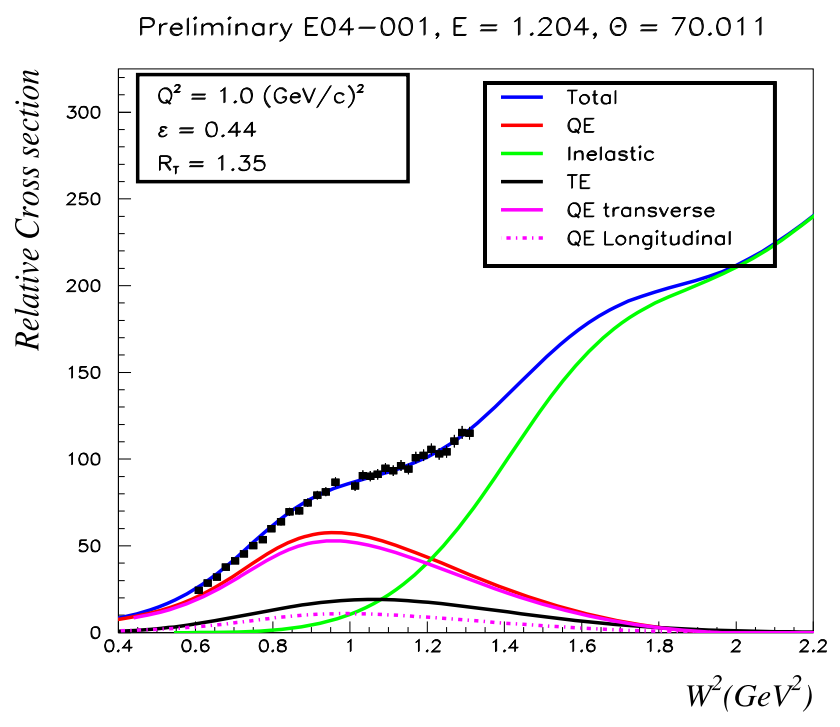

Preliminary EO4-001, E $=2.347, \Theta=30.011$

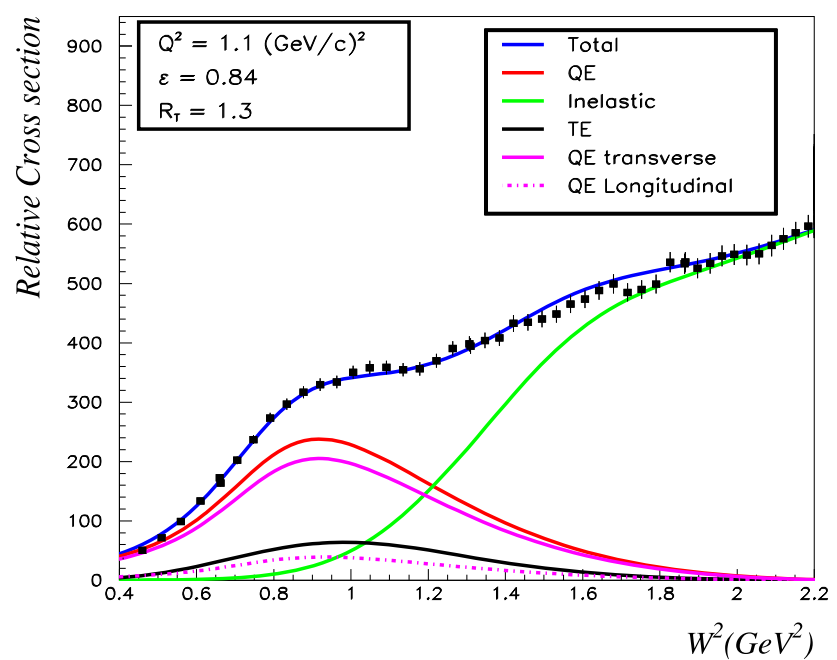

Fig. 5 Same as Fig. 4. Top: $Q^{2}=1.0 \mathrm{GeV} / \mathrm{c}^{2}$ at the QE peak. Bottom: $Q^{2}=1.1 \mathrm{GeV} / \mathrm{c}^{2}$ at the QE peak

We extract the transverse enhancement ratio as a function of $Q^{2}$ by integrating the various contributions to the fit up to $W^{2}=1.5 \mathrm{GeV}^{2}$. Here

$\mathcal{R}_{T}=\frac{\mathrm{QE}_{\text {transverse }}+\mathrm{TE}}{\mathrm{QE}_{\text {transverse }}}$

We assign a conservative systematic error to $\mathcal{R}_{T}$ to account for the possibility that a fraction of the transverse excess events may be produced with a pion in the final state.

Figure 3 shows the values of $\mathcal{R}_{T}$ as a function of $Q^{2}$. The black points are extracted from Carlson et al. [36], and the higher $Q^{2}$ blue bands are from the fit to $\mathrm{QE}$ data from the JUPITER collaboration [39]. The data are parametrized by the expression:

$\mathcal{R}_{T}=1+A Q^{2} e^{-Q^{2} / B}$ with $A=6.0$ and $B=0.34(\mathrm{GeV} / \mathrm{c})^{2}$. The electron scattering data indicate that the transverse enhancement is maximal near $Q^{2}=0.3(\mathrm{GeV} / \mathrm{c})^{2}$ and is small for $Q^{2}$ greater than $1.5(\mathrm{GeV} / \mathrm{c})^{2}$. The upper error band is given by $A=6.7$ and $B=0.35(\mathrm{GeV} / \mathrm{c})^{2}$, and the lower error band is given by $A=5.3$ and $B=0.33(\mathrm{GeV} / \mathrm{c})^{2}$. This parametrization is valid for carbon $(A=12)$ (it is also an approximate representation for higher $A$ nuclei).

\section{Consequences for $v_{\mu}, \bar{v}_{\mu}$ charged-current $\mathrm{QE}$ scattering on carbon}

We assume that there is a corresponding transverse enhancement in the $v_{\mu}, \bar{v}_{\mu}$ QE cross sections on nuclear targets. Although motivated by MEC, the analysis is model independent since the parameters are taken from electron scattering data.

In the rest of this paper, the terms cross sections and differential distributions refer to scattering from nucleons bound in carbon.

\subsection{The "Independent Nucleon $\left(M_{A}=1.014\right)$ " baseline model}

In modeling $v_{\mu}, \bar{v}_{\mu}$ QE scattering on nuclear targets we use $B B B A 2007_{25}$ free nucleon electromagnetic form factors (with $M_{V}^{2}=0.71$ ), and a dipole axial form factor with $M_{A}=1.014 \mathrm{GeV}$. We apply Pauli blocking corrections to the differential QE cross section, as implemented in the NEUGEN Monte Carlo [8-11]. The Pauli blocking factor as a function of $Q^{2}$ is shown in Fig. 6. We do not apply Fermi motion corrections since we only study the total integrated

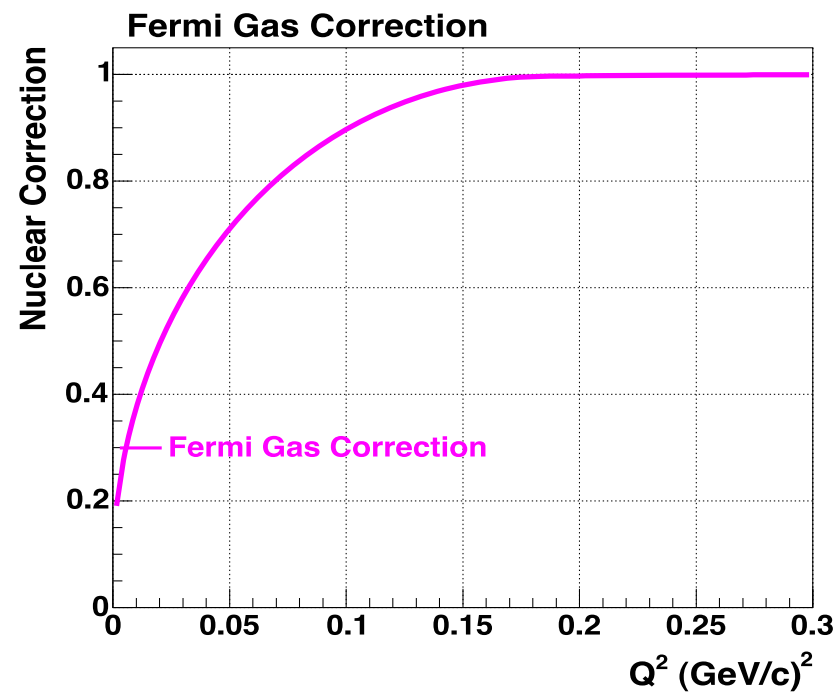

Fig. 6 The Fermi suppression factor (Pauli blocking) used in our studies as a function of $Q^{2}$. We use the Pauli blocking factor which is implemented in the NUEGEN Monte Carlo [8-11] 
QE cross section. We refer to this baseline model, which is shown as orange dotted lines on plots, as the "Independent Nucleon $\left(M_{A}=1.014\right)$ " model.

\subsection{The "Transverse Enhancement" model}

We use our parametrization of $\mathcal{R}_{T}$ to modify $G_{M p}$ and $G_{M n}$ for bound nucleons as follows. First, we assume that the enhancement in the transverse QE cross section modifies $\mathcal{G}_{M}^{V}=G_{M p}-G_{M n}$ for nucleons bound in carbon with a form given by

$$
\begin{aligned}
& G_{M p}^{\text {nuclear }}\left(Q^{2}\right)=G_{M p}\left(Q^{2}\right) \times \sqrt{1+A Q^{2} e^{-Q^{2} / B}} \\
& G_{M n}^{\text {nuclear }}\left(Q^{2}\right)=G_{M n}\left(Q^{2}\right) \times \sqrt{1+A Q^{2} e^{-Q^{2} / B}}
\end{aligned}
$$

In all of the studies we keep $G_{E p}\left(Q^{2}\right), G_{E n}\left(Q^{2}\right)$ and $F_{A}\left(Q^{2}\right)$ for bound nucleons the same as for free nucleons. The transverse enhancement leads to an enhancement in the structure functions $\mathcal{W}_{1}^{\text {Qelastic }}, \mathcal{W}_{2}^{\text {Qelastic }}$ and $\mathcal{W}_{3}^{\text {Qelastic }}$. The expressions for the $v_{\mu}, \bar{v}_{\mu}$ differential QE cross sections are given in the Appendix. We also apply Pauli blocking as a function of $Q^{2}$ as shown in Fig. 6. We refer to this model as the "Transverse Enhancement" model. The predictions on the plots for the "Transverse Enhancement Model" are shown with solid red lines. The error bands are shown as dotted dashed red lines. The ratio of calculated quantities for the "Transverse Enhancement model" divided by "Independent Nucleon" $\left(M_{A}=1.014\right)$ are also shown as solid red lines.

\subsection{The "Larger $M_{A}\left(M_{A}=1.3\right)$ " model}

Since low energy neutrino experiments have used an adhoc $M_{A}^{\text {eff }} \approx 1.3 \mathrm{GeV}$ to account for additional nuclear effects, we also compare our results to the differential and total QE cross sections calculated for independent nucleons with $M_{A}^{\text {eff }}=1.3 \mathrm{GeV}$ in the following expression:

$$
F_{A}^{\text {nuclear }}\left(Q^{2}\right)=\frac{1}{\left(1+Q^{2} / M_{A}^{2}\right)^{2}}
$$

For this model, we use the electromagnetic form factors for free nucleons, and apply Pauli blocking as described above. We refer to this model, which is shown as dashed blue lines on plots, as the "Larger $M_{A}\left(M_{A}=1.3\right)$ " model. The ratio of calculated quantities for the "Larger $M_{A}\left(M_{A}=1.3\right)$ " model divided by the predictions of the "Independent Nucleon" $\left(M_{A}=1.014\right)$ model are also shown as dashed blue lines.

\subsection{Results}

Figures 7 and 8 show the QE differential cross section $\left(\mathrm{d} \sigma / \mathrm{d} Q^{2}\right)$ as a function of $Q^{2}$ for $v_{\mu}, \bar{v}_{\mu}$ energies of 1.0
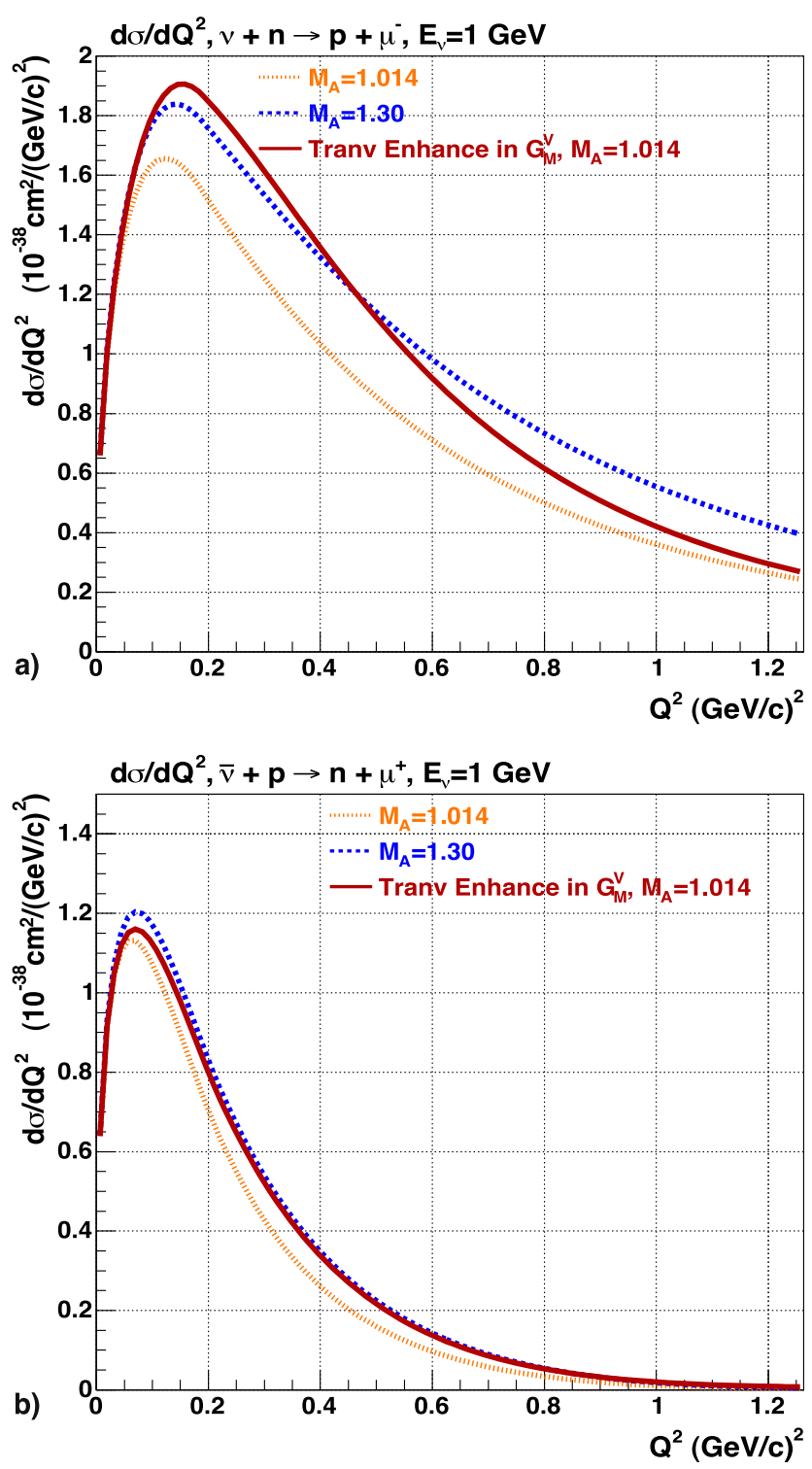

Fig. 7 The $\mathrm{QE}$ differential cross section $\left(\mathrm{d} \sigma / \mathrm{d} Q^{2}\right)$ as a function of $Q^{2}$ for $v_{\mu}, \bar{v}_{\mu}$ energies of $1.0 \mathrm{GeV}$ (maximum accessible $\left.Q_{\text {max }}^{2}=1.3(\mathrm{GeV} / \mathrm{c})^{2}\right)$. Here, the orange dotted line is the prediction of the "Independent Nucleon $\left(M_{A}=1.014\right)$ " model. The blue dashed line is the prediction of the "Larger $M_{A}\left(M_{A}=1.3\right)$ " model. The red line is prediction of the "Transverse Enhancement" model. This color and line style convention is used in all subsequent plots. Top (a): $v_{\mu}$ differential QE cross sections. Bottom $(\mathbf{b}): \bar{v}_{\mu}$ differential QE cross sections

and $3.0 \mathrm{GeV}$, respectively. The orange dotted line is the prediction of the "Independent Nucleon $\left(M_{A}=1.014\right)$ " model, the blue dashed line is the prediction of the "Larger $M_{A}$ $\left(M_{A}=1.3\right)$ " model, and the solid red line is the prediction of the "Transverse Enhancement" model. The top panels (a) show $v_{\mu}$ differential QE cross sections, and the bottom panels (b) show the $\bar{v}_{\mu}$ differential QE cross sections.

Figures 9 and 10 show the ratio of the predictions of the two models to the predictions of the "Independent Nucleon 

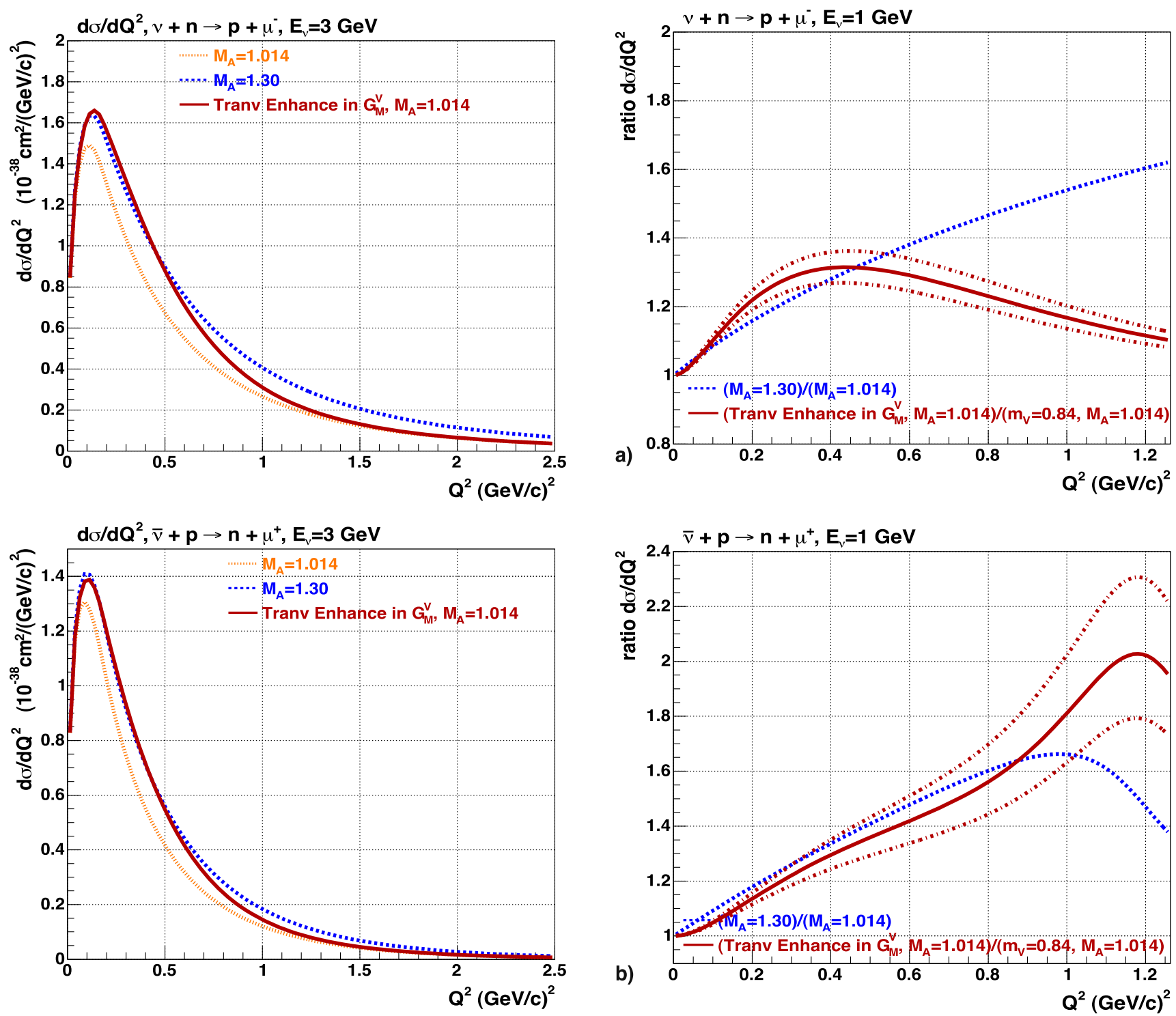

Fig. 8 Same as Fig. 7 for $v_{\mu}, \bar{v}_{\mu}$ energies of $3.0 \mathrm{GeV}$ (maximum accessible $\left.Q_{\max }^{2}=4.9(\mathrm{GeV} / \mathrm{c})^{2}\right)$

$\left(M_{A}=1.014\right)$ " model as a function of $Q^{2}$ for $v_{\mu}, \bar{v}_{\mu}$ energies of $1.0 \mathrm{GeV}$, and $3.0 \mathrm{GeV}$, respectively. The blue dashed line is the ratio for the "Larger $M_{A}\left(M_{A}=1.3\right)$ " model. The red line is the ratio for the "Transverse Enhancement" mode (with error bands shown as dotted red lines). The top (a)

Fig. 9 The ratio of the prediction of the two models for the QE differential cross section $\mathrm{d} \sigma / \mathrm{d} Q^{2}$ to the prediction of the "Independent Nucleon $\left(M_{A}=1.014\right)$ " model as a function of $Q^{2}$ for $v_{\mu}, \bar{v}_{\mu}$ energies of $1.0 \mathrm{GeV}$ (maximum accessible $\left.Q_{\max }^{2}=1.3(\mathrm{GeV} / \mathrm{c})^{2}\right)$. The blue dashed line is the ratio for the "Larger $M_{A}\left(M_{A}=1.3\right)$ " model. The red line is the ratio for the "Transverse Enhancement" model (with error bands shown as dotted red lines). Top (a): ratio for $v_{\mu}$ differential QE cross sections. Middle (b): ratio for $\bar{v}_{\mu}$ differential QE cross sections. Bottom (c): The $\bar{v}_{\mu} / v_{\mu}$ ratio for the differential QE cross sections divided by the corresponding $\bar{v}_{\mu} / v_{\mu}$ ratio for the "Independent Nucleon $\left(M_{A}=1.014\right)$ " model

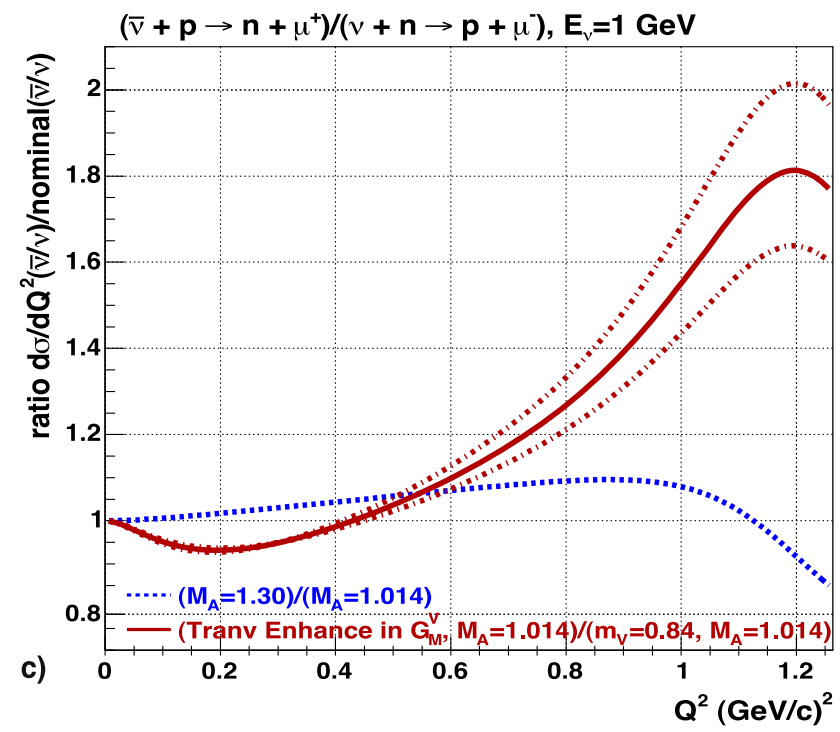



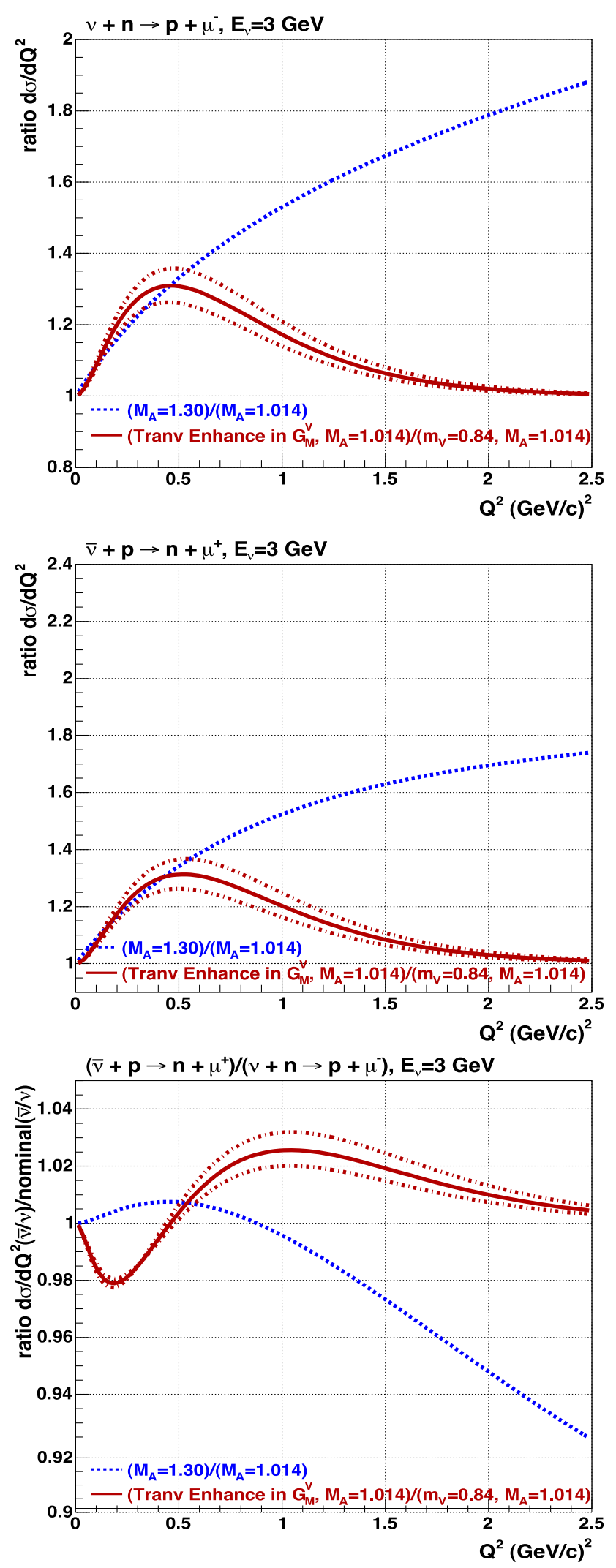

Fig. 10 Same as Fig. 9 for $v_{\mu}, \bar{v}_{\mu}$ energies of $3.0 \mathrm{GeV}$ (maximum accessible $\left.Q_{\max }^{2}=4.9(\mathrm{GeV} / \mathrm{c})^{2}\right)$ panels show the ratio for $\mathrm{d} \sigma / \mathrm{d} Q^{2}$ for $v_{\mu}$. The middle (b) panels show the ratio for $\mathrm{d} \sigma / d Q^{2}$ for $\bar{v}_{\mu}$. The bottom (c) panels show the ratio of predicted ratio of $\bar{v}_{\mu} / \nu_{\mu} \mathrm{d} \sigma / \mathrm{d} Q^{2}$ cross sections for the two models (divided by the $\bar{v}_{\mu} / v_{\mu}$ ratio predicted by the "Independent Nucleon $\left(M_{A}=1.014\right)$ " model).

For $Q^{2}<0.6(\mathrm{GeV} / \mathrm{c})^{2}$ the differential $\mathrm{QE}$ cross section for the "Transverse Enhancement" model is close to the "Larger $M_{A}\left(M_{A}=1.3\right)$ " model. The maximum accessible $Q^{2}$ for $1 \mathrm{GeV}$ neutrinos is $1.3(\mathrm{GeV} / \mathrm{c})^{2}$ (as shown in Fig. 16). Therefore, fits to the neutrino differential QE cross sections for an incident energy of $1 \mathrm{GeV}$ (e.g. MiniBooNE) would yield $M_{A} \approx 1.2 \mathrm{GeV}$. The extracted value of $M_{A}$ depends on the specific model parameters that are used for Pauli blocking and the variation of the statistical errors in the data with $Q^{2}$. For a neutrino energy of $1 \mathrm{GeV}$, the total integrated QE cross section predicted by the "Transverse Enhancement" model is also larger than the total QE cross section prediction of the "Independent Nucleon $\left(M_{A}=1.014\right)$ " model.

In the high $Q^{2}$ region $\left(Q^{2}>1.2(\mathrm{GeV} / \mathrm{c})^{2}\right)$, the predicted differential QE cross section for the "Transverse Enhancement" model is similar to the prediction of the "Independent Nucleon $\left(M_{A}=1.014\right)$ " model. The maximum accessible $Q^{2}$ for $3 \mathrm{GeV}$ neutrinos is $4.9(\mathrm{GeV} / \mathrm{c})^{2}$. In order to reduce the sensitivity to modeling of Pauli blocking, experiments at higher energy [26, 27] typically remove the lower $Q^{2}$ points in fits for $M_{A}$. Consequently, fits for the neutrino differential QE cross sections measured in high energy experiments would yield a value of $M_{A}$ which is smaller than $1.014 \mathrm{GeV}$ because for $Q^{2}>0.5(\mathrm{GeV} / \mathrm{c})^{2}$ the slope of the differential $\mathrm{QE}$ cross section in the transition region between low and high $Q^{2}$ is steeper than for $M_{A}=1.014 \mathrm{GeV}$. This is consistent with the fact that the average $M_{A}$ extracted from high energy data on nuclear targets $[26,27]$ is $0.979 \pm 0.016$.

Figure 11 shows the total QE cross section as function of energy. The data points are the measurements from MiniBooNE [13, 14] and NOMAD [30, 31]. The orange dotted line is the prediction of the "Independent Nucleon $\left(M_{A}=1.014\right)$ " model. The blue dashed line is prediction of the "Larger $M_{A}\left(M_{A}=1.3\right)$ ". The red line is the prediction of the "Transverse Enhancement"' model (with error bands shown as dotted red lines). The top (a) panel shows the $v_{\mu}$ total QE cross section. The middle (b) panel shows the $\bar{v}_{\mu}$ total $\mathrm{QE}$ cross section. The bottom (c) panel shows the ratio of $\bar{v}_{\mu}$ and $v_{\mu}$ total QE cross sections.

Figure 12 shows the ratio of the predictions for total QE cross section to the predictions of the "Independent $\mathrm{Nu}$ cleon $\left(M_{A}=1.014\right)$ " model as a function energy. The blue dashed line is the ratio of the predictions for the "Larger $M_{A}\left(M_{A}=1.3\right)$ " model, and the red line is ratio for the "Transverse Enhancement" model (with error bands shown as dotted red lines). The top (a) panel shows the ratio of the 

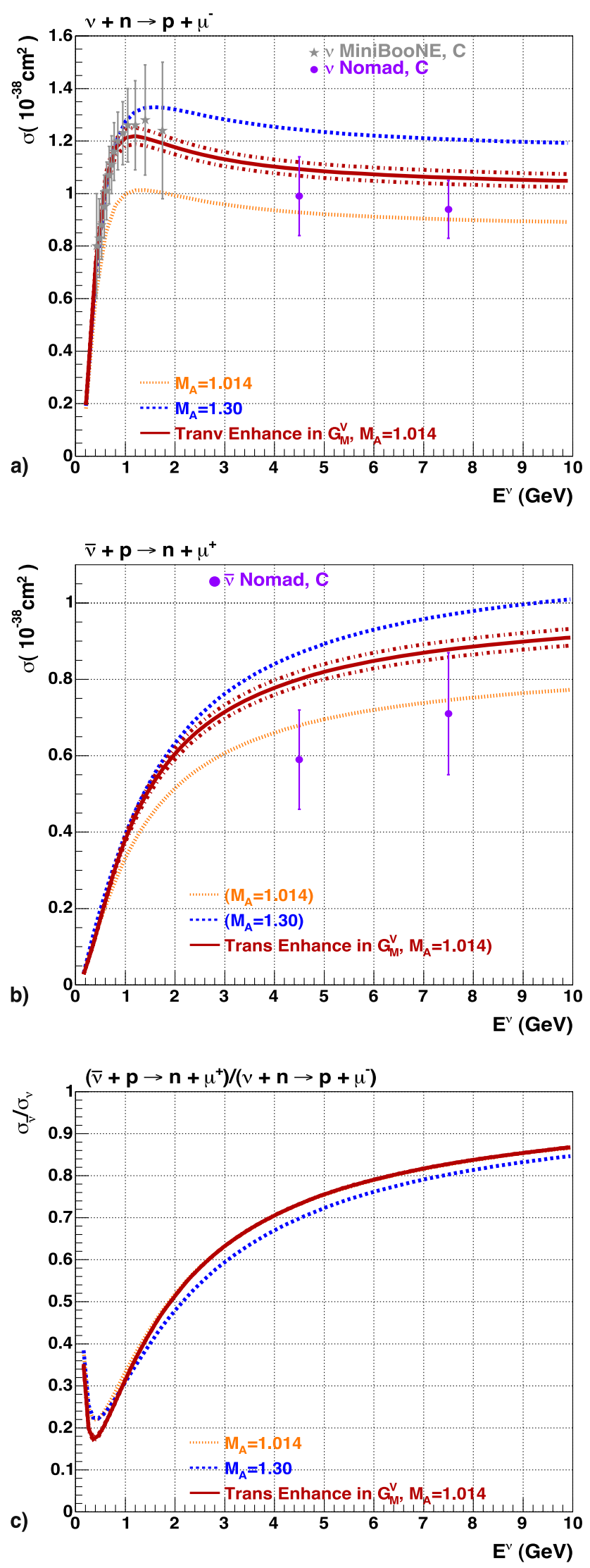

Fig. 11 The total QE cross section as function of energy. The data points are measurements of MiniBooNE [13, 14] (gray stars) and NOMAD $[30,31]$ (purple circles). The predictions for the "Independent Nucleon $\left(M_{A}=1.014\right)$ " model, "Larger $M_{A}\left(M_{A}=1.3\right)$ model", and "Transverse Enhancement model" are shown. Top (a): $v_{\mu}$ total QE cross section. Middle (b): $\bar{v}_{\mu}$ total QE cross section. Bottom (c): QE $\bar{v}_{\mu} / v_{\mu}$ total cross section ratio

predictions for the $v_{\mu}$ total QE cross section. The middle (b) panel shows the ratio of the predictions for the $\bar{v}_{\mu}$ total QE cross section. The bottom (c) panel shows the predicted $\bar{v}_{\mu} / v_{\mu}$ cross section ratio divided by the predicted $\bar{v}_{\mu} / v_{\mu}$ ratio for the "Independent Nucleon $\left(M_{A}=1.014\right)$ " model. The data points are measurements from MiniBooNE $[13,14]$ and NOMAD [30, 31].

As shown in Fig. 11(a) (top), and Fig. 12(a) (top), at low $v_{\mu}$ energies the "Transverse Enhancement" model (red line) predicts QE cross sections at a level similar to the "Larger $M_{A}\left(M_{A}=1.3\right)$ " model (blue dashed line). Both the "Larger $M_{A}\left(M_{=} 1.3\right)$ " model and the "Transverse Enhancement" model predictions are in agreement with the MiniBooNE QE $v_{\mu}$ cross sections. However, at higher $v_{\mu}$ energies the "Transverse Enhancement" model predicts QE cross sections which are lower than the prediction of the "Larger $M_{A}$ $\left(M_{A}=1.3\right)$ ". The lower $\mathrm{QE} v_{\mu}$ cross sections at high energy are consistent with the NOMAD measurements (within experimental errors).

Similarly, for $\bar{v}_{\mu}$ scattering the "Transverse Enhancement" model predicts total QE cross section which are lower than the predictions of the "Larger $M_{A}\left(M_{A}=1.3\right)$ " model as shown in Fig. 11(b) (middle) and Fig. 12(b) (middle). The lower QE cross $\bar{v}_{\mu}$ sections are consistent with the NOMAD measurements (within experimental errors).

\section{Conclusion}

We parametrize the enhancement in the transverse QE cross section observed in QE electron scattering on nuclear targets as a correction to the magnetic form factors of bound nucleons. Within models of MEC, MEC processes contribute only to the transverse QE response function and do not enhance the longitudinal and axial response functions. We find that the $\mathrm{QE}$ cross sections for $v_{\mu}, \bar{v}_{\mu} \mathrm{QE}$ scattering predicted by the "Transverse Enhancement" model agree with the MiniBooNE low energy neutrino QE cross sections, and are also consistent with QE cross sections measured by NOMAD at higher energies.

The simple two parameter parametrization of $Q^{2}$ dependence of the transverse enhancement as a correction to the proton and neutron magnetic form factors can easily be incorporated into existing Monte Carlo generators [8-11].

At present, $v_{\mu}, \bar{v}_{\mu}$ experiments use the "Large $M_{A}$ " model to predict $\mathcal{W}_{1}^{\text {Qelastic }}, \mathcal{W}_{2}^{\text {Qelastic }}$, and $\mathcal{W}_{3}^{\text {Qelastic }}$ for neu- 

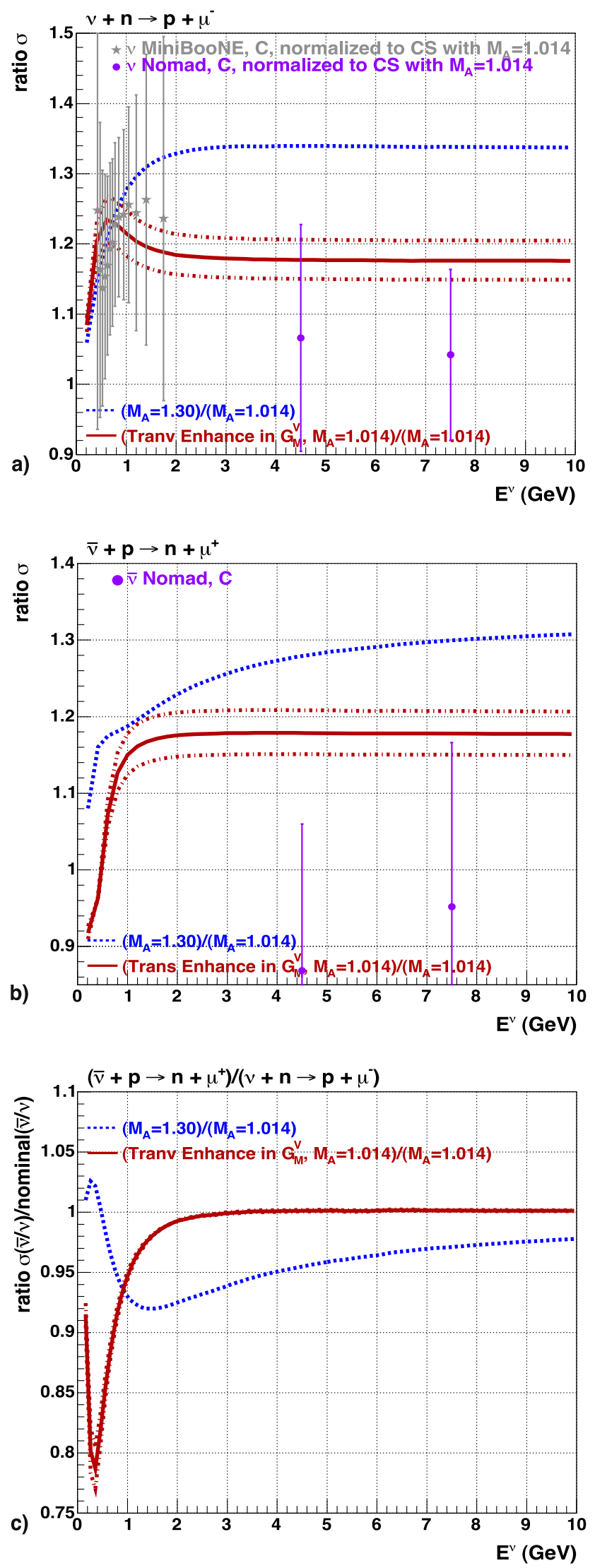

4 Fig. 12 The ratios of predicted and measured total QE cross section to the predictions of the "Independent Nucleon $\left(M_{A}=1.014\right)$ " model as a function energy. The ratios for the predictions of the "Larger $M_{A}$ $\left(M_{A}=1.3\right)$ model" and "Transverse Enhancement model" are shown. The data points are the ratios for the measurements of MiniBooNE [13, 14] (gray stars) and NOMAD [30, 31] (purple circles). Top (a): The ratio for $v_{\mu}$ total QE cross sections. Middle (b): The ratio for $\bar{v}_{\mu} \mathrm{QE}$ cross sections. Bottom (c): The $\bar{v}_{\mu} / v_{\mu}$ total QE cross section ratio divided by the corresponding ratio for the "Independent Nucleon $\left(M_{A}=1.014\right)$ " model

trino QE scattering on nuclear targets. A large increase in $M_{A}$ is contrary to theoretical expectations [26-28].

The differential and total QE cross sections predicted in the "Larger $M_{A}\left(M_{A}=1.3\right)$ " model are similar to the predictions of "Transverse Enhancement" model only at low $v_{\mu}$ energies.

The assumption made in the "Transverse Enhancement" model is that the enhancements in the transverse response functions in $\bar{v}_{\mu} / v_{\mu}$ scattering are the same as measured in electron scattering, and that there is no additional enhancement in the longitudinal or axial response functions (as expected in MEC models [36]). Since we only uses parameters from electron scattering data, our analysis is purely phenomenological, and does not rely on a specific MEC model. Because in electron scattering the transverse enhancement is only significant at low values of $Q^{2}$, the fractional contribution of "Transverse Enhancement" to the total neutrino QE cross section is energy dependent, thus resolving much of the apparent discrepancy between the low energy and high energy neutrino QE cross sections on nuclear targets.

In an earlier publication, Martini, Ericson, Chanfray, and Marteau [37, 38] calculated the contribution of meson exchange currents to the differential and total QE cross sections for $\bar{v}_{\mu} / v_{\mu}$ energies less than $1.2 \mathrm{GeV}$. In the comparison with our model, we show the Martini et al. predictions with the random phase approximation ("QE+np-nh RPA"). For the range $0.5<E<1.2 \mathrm{GeV}$, the predictions of Martini et al. are similar to the predictions of the "Transverse Enhancement" model as shown in Figs. 13, and 14. For $E<0.5$, the predictions of Martini et al. are lower than the predictions of the "Transverse Enhancement" model. However, for such low energies, the predictions are sensitive to differences in the modeling of Pauli blocking in the two models. The predictions of the Martini et al. model for $\bar{v}_{\mu} / v_{\mu}$ scattering for energies greater than $1.2 \mathrm{GeV}$ have not yet been published.

Figure 15 shows a comparison of the various model for a larger energy range ( 0.1 to $100 \mathrm{GeV})$. The energy dependence for the predictions of the transverse enhancement model originates from the energy dependence of the maximum accessible $Q^{2}\left(Q_{\max }^{2}\right)$ for QE scattering, as shown in Fig.16. The lower energies have lower $Q_{\max }^{2}$ where the transverse enhancement is large, while higher energies have a higher $Q_{\text {max }}^{2}$, where the transverse enhancement is small. 

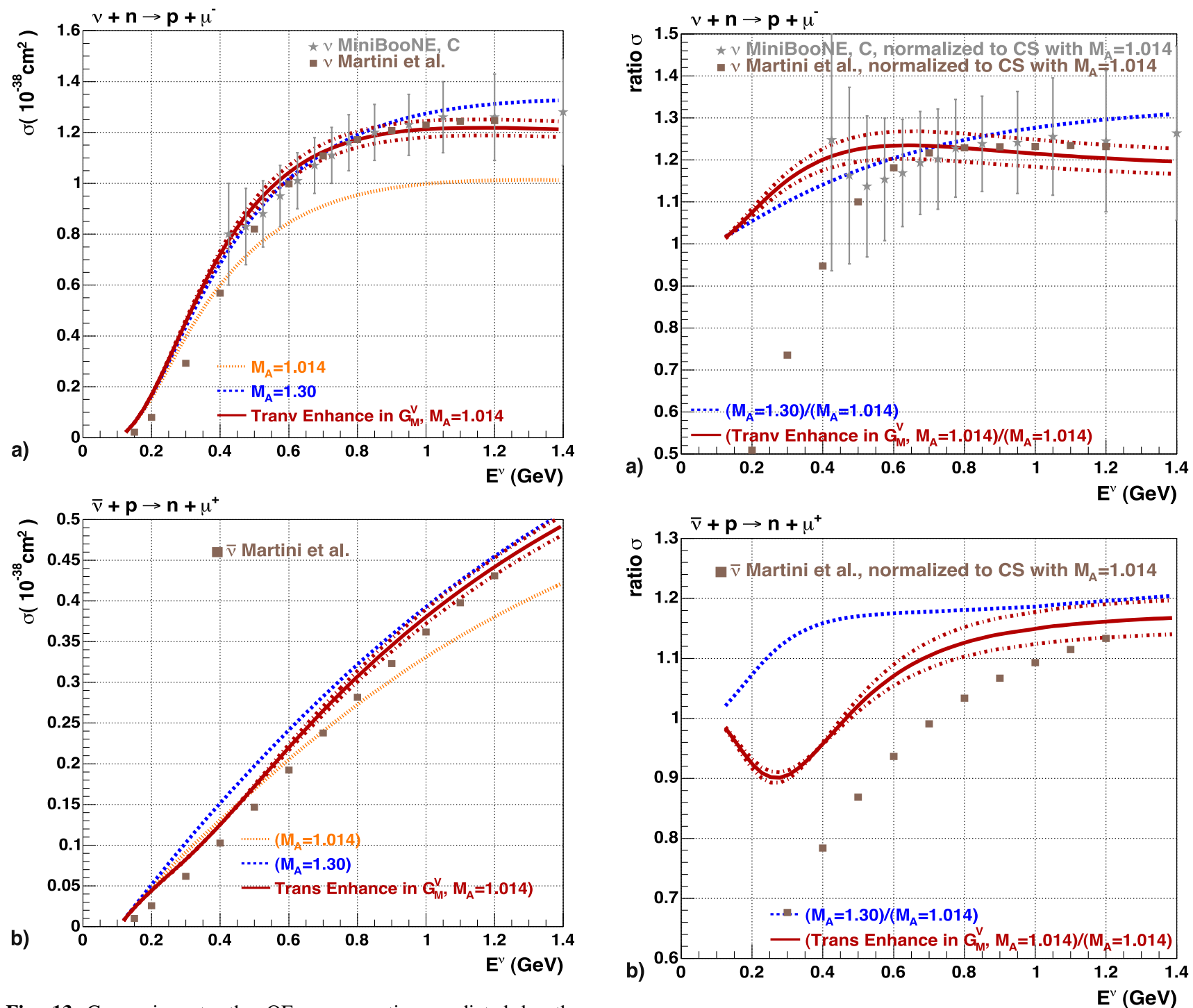

Fig. 13 Comparison to the $Q E$ cross section predicted by the "QE+np-nh RPA" MEC model of Martini et al. [37, 38] (predictions for this model have only been published for neutrino energies less than $1.2 \mathrm{GeV})$. The predictions for the "Independent Nucleon $\left(M_{A}=1.014\right)$ " model, "Larger $M_{A}\left(M_{A}=1.3\right)$ model", and "Transverse Enhancement model" are shown. The gray squares are the predictions of the MEC model of Martini et al. [37, 38]. The data points are measurements from MiniBooNE [13, 14] (gray stars). Top (a): $v_{\mu}$ total QE cross section. Middle (b): $\bar{v}_{\mu}$ total QE cross section

The differential cross section at high energy is almost independent of energy, as shown in Figs. 17, 18, 19 and 20.

\section{Testing the model with neutrino data}

The MINERvA high statistics neutrino experiment [51] at Fermilab is currently taking data with a fully active scintillator target calorimeter in the NUMI beam (with both neutrinos and antineutrinos). The QE differential cross sections would be measured as a function of $Q^{2}$ at a variety of neu-

Fig. 14 Comparison to the QE cross section predicted by the "QE+np-nh RPA" MEC model of Martini et al. [37, 38] (predictions for this model have only been published for neutrino energies less than $1.2 \mathrm{GeV})$. Shown are the ratios for the predictions of the "Larger $M_{A}$ $\left(M_{A}=1.3\right)$ model" and "Transverse Enhancement model" to the "Independent Nucleon $\left(M_{A}=1.014\right)$ " QE cross section as a function energy. The gray squares are the ratios for the predictions of the MEC model of Martini et al. [37, 38]. The data points are the ratios for the measurements of MiniBooNE [13, 14] (gray stars). Top (a): The ratio for $v_{\mu}$ total QE cross sections. Bottom (b): The ratio for $\bar{v}_{\mu}$ QE cross sections

trino energies within one single experiment, and compared to the predictions of various models.

Open Access This article is distributed under the terms of the Creative Commons Attribution Noncommercial License which permits any noncommercial use, distribution, and reproduction in any medium, provided the original author(s) and source are credited. 

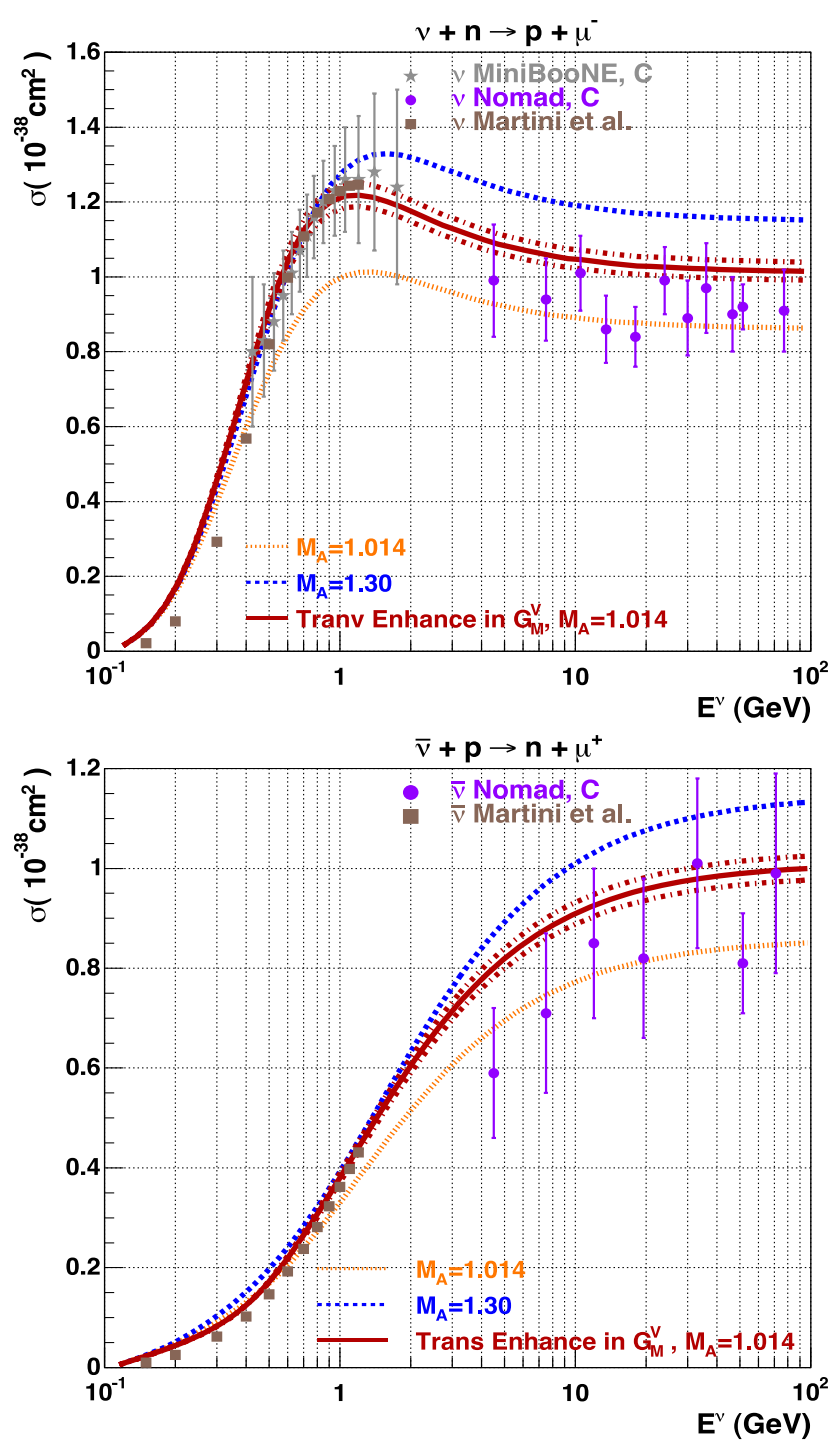

Fig. 15 Comparison of predictions for the $v_{\mu}, \bar{v}_{\mu}$ total QE cross section at high energies for the "Independent Nucleon $\left(M_{A}=1.014\right)$ " model, the "Larger $M_{A}\left(M_{A}=1.3\right)$ model", the "Transverse Enhancement model", and the "QE+np-nh RPA" MEC model of Martini et al. $[37,38]$ (predictions for this model have only been published for neutrino energies less than $1.2 \mathrm{GeV}$ ). The data points are the ratios for the measurements of MiniBooNE [13, 14] (gray stars) and NOMAD [30, 31] (purple circles)

\section{Appendix: $v_{\mu}, \bar{v}_{\mu}$ nucleon/nucleus scattering}

At a fixed value of the final state invariant mass $W$, the differential cross section for $v_{\mu}, \bar{v}_{\mu}$ scattering at incident en$\operatorname{ergy} E$ is given [44] by:

$$
\begin{aligned}
\frac{d \sigma}{d Q^{2} d W}= & \frac{G^{2}}{2 \pi} \cos ^{2} \theta_{C} \frac{W}{M}\left\{\frac{1}{2 E^{2}} \mathcal{W}_{1}\left[Q^{2}+m_{\mu}^{2}\right]\right. \\
& +\mathcal{W}_{2}+\mathcal{W}_{2}\left[-\frac{v}{E}-\frac{1}{4 E^{2}}\left(Q^{2}+m_{\mu}^{2}\right)\right]
\end{aligned}
$$

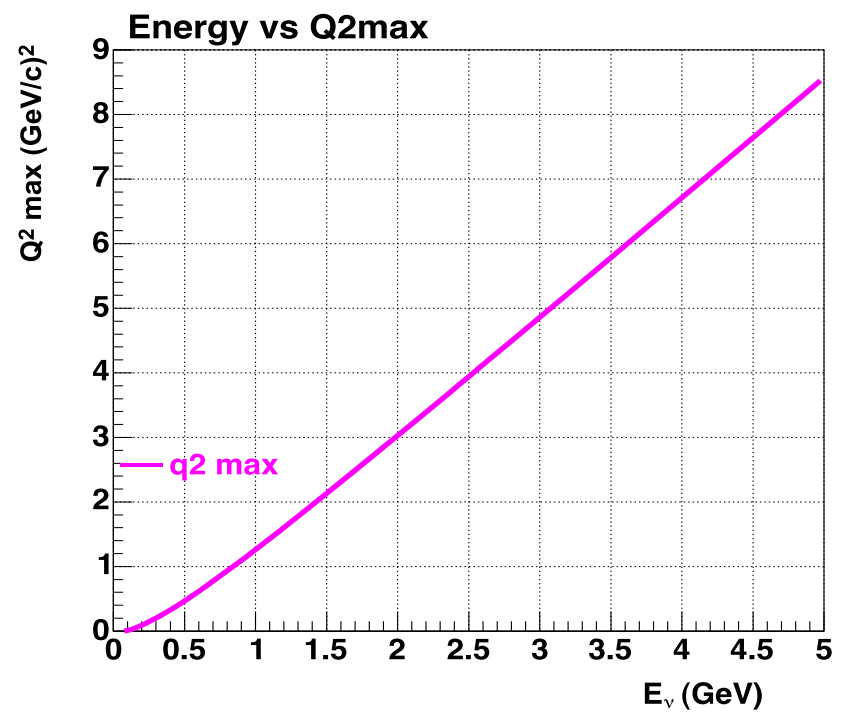

Fig. 16 The maximum accessible $Q^{2}$ for $\mathrm{QE}$ events as a function of neutrino energy

$$
\left.\begin{array}{l} 
\pm \mathcal{W}_{3}\left[\frac{Q^{2}}{2 M E}-\frac{v}{4 E} \frac{Q^{2}+m_{\mu}^{2}}{M E}\right] \\
+\frac{\mathcal{W}_{4}}{M^{2}} m_{\mu}^{2} \frac{\left(Q^{2}+m_{\mu}^{2}\right)}{4 E^{2}}-\frac{\mathcal{W}_{5}}{M E} m_{\mu}^{2}
\end{array}\right\}
$$

Here, $\frac{G^{2}}{2 \pi} \cos ^{2} \theta_{C}=80 \times 10^{-40} \mathrm{~cm}^{2} / \mathrm{GeV}^{2}$. The final state muon mass places the following kinematic limits [45-47] on $x=Q^{2} / 2 M v$ and $y=v / E$ :

$\frac{m_{\mu}^{2}}{2 M\left(E_{v}-m_{\mu}\right)} \leq x \leq 1$
$a-b \leq y \leq a+b$

where the quantities $a$ and $b$ are

$a=\left[1-m_{\mu}^{2}\left(\frac{1}{2 M E_{v} x}+\frac{1}{2 E_{v}^{2}}\right)\right] /\left(2+M x / E_{v}\right)$

$b=\left[\left(1-\frac{m_{\mu}^{2}}{2 M E_{v} x}\right)^{2}-\frac{m_{\mu}^{2}}{E_{v}^{2}}\right]^{1 / 2} /\left(2+M x / E_{v}\right)$

Or alternatively, for a fixed energy and $Q^{2}$, there is a maximum value of $W$ which is given by [48]:

$$
\begin{aligned}
W_{+}^{2}\left(Q^{2}\right)= & {\left[\frac{1}{4} s^{2} a_{-}^{2}\left(\frac{m_{\mu}^{4}}{s^{2}}-2 \frac{m_{\mu}^{2}}{s}\right)-\left(Q^{2}+\frac{1}{2} m_{\mu}^{2} a_{+}^{2}\right)^{2}\right.} \\
& \left.+s a_{-}\left(Q^{2}+\frac{m_{\mu}^{2}}{2} a_{+}\right)\right] /\left[a_{-}\left(Q^{2}+m_{\mu}^{2}\right)\right]
\end{aligned}
$$

where $s=2 M E+M^{2}, a_{ \pm}=1 \pm M^{2} / s$. For QE scattering, this corresponds to a minimum and maximum accessible $Q^{2}$ for a given neutrino energy. The maximum accessible $Q^{2}$ 

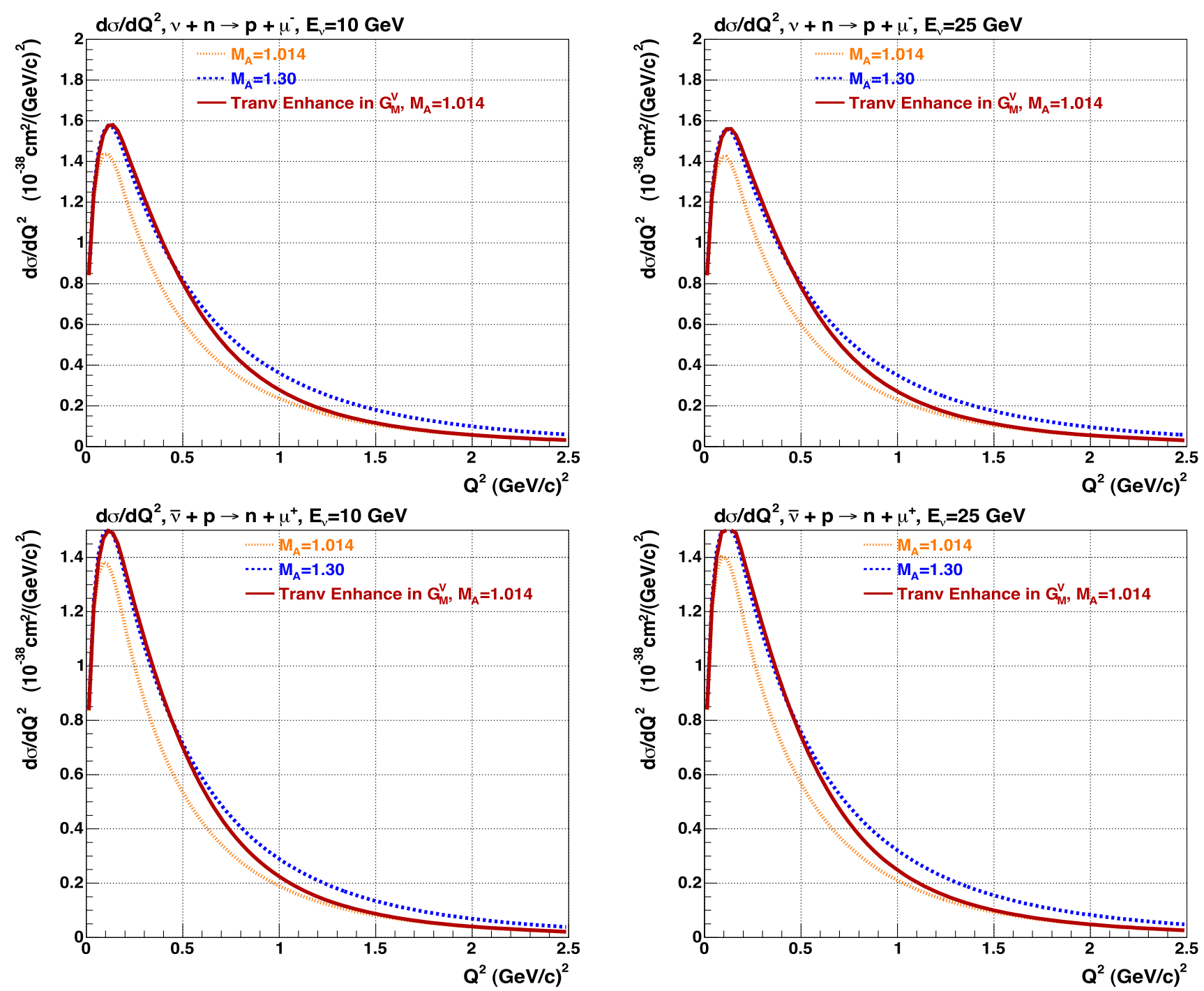

Fig. 17 Same as Fig. 7 for $v_{\mu}, \bar{v}_{\mu}$ energies of $10.0 \mathrm{GeV}$

Fig. 18 Same as Fig. 7 for $v_{\mu}, \bar{v}_{\mu}$ energies of $25.0 \mathrm{GeV}$

$\left(Q_{\max }^{2}\right)$ for $\mathrm{QE}$ events as a function of neutrino energy is shown in Fig. 16.

\section{A.1 Quasielastic $v_{\mu}, \bar{v}_{\mu}$ scattering}

A theoretical framework for quasi-elastic $\left(v_{\mu}, \bar{v}_{\mu}\right)$-Nucleon Scattering has been given by Llewellyn Smith [49, 50]. Here, we use the notation of Llewellyn Smith (except that $F_{V}^{2}$ in our notation is equal to $\xi_{l s} F_{V}^{2}$ in Llewellyn Smith's notation, where $\left.\xi_{l s}=\left(\mu_{p}-1-\mu_{n}\right)\right)$. In addition, we use $Q^{2}$ while Llewellyn Smith uses $q^{2}$ where

$q^{2}=q_{0}^{2}-\mathbf{q}_{3}^{2}=-4 E_{0} E^{\prime} \sin ^{2} \frac{\theta}{2}=-Q^{2}$

The hadronic current for $\mathrm{QE} v_{\mu}, \bar{v}_{\mu}$ scattering is given by $[49,50]$

$\left\langle p\left(p_{2}\right)\left|J_{\lambda}^{+}\right| n\left(p_{1}\right)\right\rangle$

$$
\begin{aligned}
= & \bar{u}\left(p_{2}\right)\left[\gamma_{\lambda} \mathcal{F}_{1}^{V}\left(q^{2}\right)+\frac{i \sigma_{\lambda \nu} q^{\nu} \mathcal{F}_{2}^{V}\left(q^{2}\right)}{2 M}\right. \\
& \left.+\gamma_{\lambda} \gamma_{5} \mathcal{F}_{A}\left(q^{2}\right)+\frac{q_{\lambda} \gamma_{5} \mathcal{F}_{P}\left(q^{2}\right)}{M}\right] u\left(p_{1}\right)
\end{aligned}
$$

where $q=k_{v}-k_{\mu}$, and $M=\left(m_{p}+m_{n}\right) / 2$. Here, $\mu_{p}$ and $\mu_{n}$ are the proton and neutron magnetic moments. We assume that there are no second class currents, so the scalar form factor $\mathcal{F}_{V}^{3}$ and the tensor form factor $\mathcal{F}_{A}^{3}$ need not be included. Using the above current, the QE cross section is

$$
\begin{aligned}
\frac{d \sigma^{\nu, \bar{v}}}{d Q^{2}}= & \frac{M^{2} G_{F}^{2} \cos ^{2} \theta_{c}}{8 \pi E_{v}^{2}} \\
& \times\left[A\left(Q^{2}\right) \mp \frac{(s-u) B\left(Q^{2}\right)}{M^{2}}+\frac{C\left(Q^{2}\right)(s-u)^{2}}{M^{4}}\right]
\end{aligned}
$$



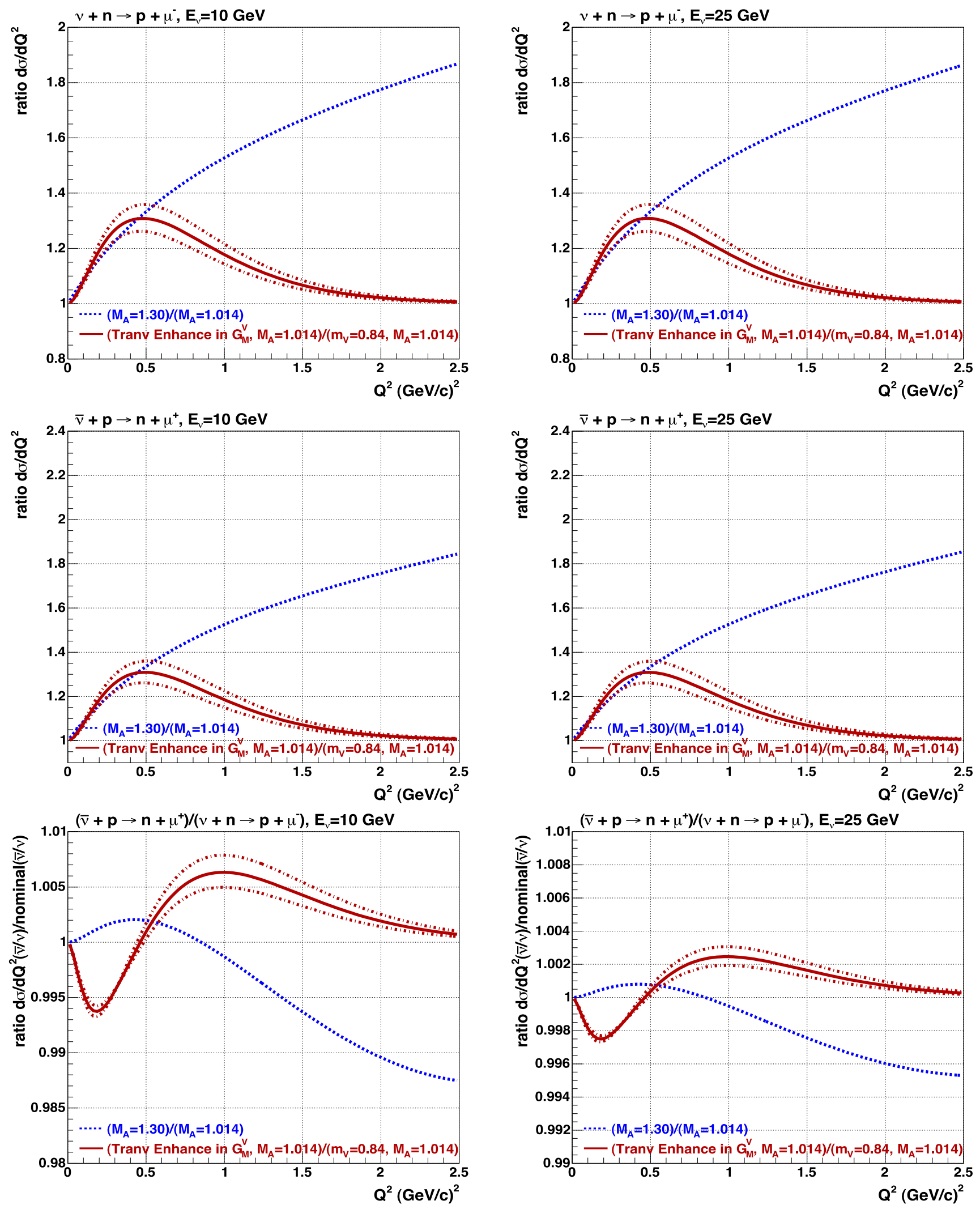

Fig. 19 Same as Fig. 9 for $v_{\mu}, \bar{v}_{\mu}$ energies of $10 \mathrm{GeV}$

Fig. 20 Same as Fig. 9 for $v_{\mu}, \bar{v}_{\mu}$ energies of $25 \mathrm{GeV}$ 
where $s-u=4 M E_{\nu}-Q^{2}-m_{\mu}^{2}$.

$$
\begin{aligned}
A\left(Q^{2}\right)= & \frac{m_{\mu}^{2}+Q^{2}}{M^{2}}\left\{(1+\tau)\left|\mathcal{F}_{A}\right|^{2}-(1-\tau)\left|\mathcal{F}_{1}^{V}\right|^{2}\right. \\
& \left.+\tau(1-\tau)\left|\mathcal{F}_{2}^{V}\right|^{2}+4 \tau \mathcal{F}_{1}^{V} \mathcal{F}_{2}^{V}\right\} \\
& -\frac{m_{\mu}^{2}+Q^{2}}{M^{2}} \frac{m_{\mu}^{2}}{4 M^{2}}\left\{\left(\left|\mathcal{F}_{1}^{V}+\mathcal{F}_{2}^{V}\right|^{2}\right)\right. \\
& \left.+\left(\mathcal{F}_{A}+2 \mathcal{F}_{P}\right)^{2}-4(1+\tau) \mathcal{F}_{P}^{2}\right\} \\
B\left(Q^{2}\right)= & 4 \tau \mathcal{F}_{A}\left(\mathcal{F}_{1}^{V}+\mathcal{F}_{2}^{V}\right)=4 \tau \mathcal{F}_{A} \mathcal{G}_{M}^{V} \\
C\left(Q^{2}\right)= & \frac{1}{4}\left(\left|\mathcal{F}_{A}\right|^{2}+\left|\mathcal{F}_{1}^{V}\right|^{2}+\tau\left|\mathcal{F}_{2}^{V}\right|^{2}\right) \\
= & \frac{1}{4}\left(\left|\mathcal{F}_{A}\right|^{2}+\left|F_{V}\left(Q^{2}\right)\right|^{2}\right)
\end{aligned}
$$

Where $\tau=Q^{2} / 4 M^{2}$. The form factors $F_{1}^{V}\left(Q^{2}\right)$ and $F_{2}^{V}\left(Q^{2}\right)$ are given by:

$$
\begin{aligned}
& \mathcal{F}_{1}^{V}\left(Q^{2}\right)=\frac{\mathcal{G}_{E}^{V}\left(Q^{2}\right)+\frac{Q^{2}}{4 M^{2}} \mathcal{G}_{M}^{V}\left(Q^{2}\right)}{1+\frac{Q^{2}}{4 M^{2}}} \\
& \mathcal{F}_{2}^{V}\left(Q^{2}\right)=\frac{\mathcal{G}_{M}^{V}\left(Q^{2}\right)-\mathcal{G}_{E}^{V}\left(Q^{2}\right)}{1+\frac{Q^{2}}{4 M^{2}}}
\end{aligned}
$$

From conserved vector current $(\mathrm{CVC}) \mathcal{G}_{E}^{V}\left(Q^{2}\right)$ and $\mathcal{G}_{M}^{V}\left(Q^{2}\right)$ are related to the electron scattering form factors $G_{E}^{p}\left(Q^{2}\right)$, $G_{E}^{n}\left(Q^{2}\right), G_{M}^{p}\left(Q^{2}\right)$, and $G_{M}^{n}\left(Q^{2}\right)$ :

$\mathcal{G}_{E}^{V}\left(Q^{2}\right)=G_{E}^{p}\left(Q^{2}\right)-G_{E}^{n}\left(Q^{2}\right)$

$\mathcal{G}_{M}^{V}\left(Q^{2}\right)=G_{M}^{p}\left(Q^{2}\right)-G_{M}^{n}\left(Q^{2}\right)$

We also define

$$
\left|\mathcal{F}_{V}\left(Q^{2}\right)\right|^{2}=\frac{\left[\mathcal{G}_{E}^{V}\left(Q^{2}\right)\right]^{2}+\tau\left[\mathcal{G}_{M}^{V}\left(Q^{2}\right)\right]^{2}}{1+\tau}
$$

The axial form factor $\mathcal{F}_{A}$ can be approximated by the dipole form

$$
\mathcal{F}_{A}\left(q^{2}\right)=\frac{g_{A}}{\left(1+\frac{Q^{2}}{M_{A}^{2}}\right)^{2}}
$$

Where $g_{A}=-1.267$.

The pseudoscalar form factor $\mathcal{F}_{P}$ is related to $\mathcal{F}_{A}$ by PCAC and is given by:

$$
\mathcal{F}_{P}\left(q^{2}\right)=\frac{2 M^{2} \mathcal{F}_{A}\left(q^{2}\right)}{M_{\pi}^{2}+Q^{2}}
$$

In the expression for the QE cross section, $\mathcal{F}_{P}\left(q^{2}\right)$ is multiplied by $\left(m_{\mu} / M\right)^{2}$. Therefore, in $v_{\mu}, \bar{v}_{\mu}$ interactions, this effect is very small except at very low energy, below $0.2 \mathrm{GeV}$.
In the dipole approximation,

$\mathcal{G}_{M}^{V}\left(Q^{2}\right) \approx 4.706 G_{D}^{V}\left(Q^{2}\right)$

In our analysis we apply $B B B A 2007_{25}$ corrections [19] to the dipole parametrization of the electromagnetic form factors as described in [19].

By comparing (A.1) and (A.4) and using the following expressions:

$\mathcal{F}_{1}^{V}\left(Q^{2}\right)+F_{2}^{V}\left(Q^{2}\right)=\mathcal{G}_{M}^{V}\left(Q^{2}\right)$

$\left|\mathcal{F}_{1}^{V}\left(Q^{2}\right)\right|^{2}+\tau\left|\mathcal{F}_{2}^{V}\left(Q^{2}\right)\right|^{2}=\left|\mathcal{F}_{V}\left(Q^{2}\right)\right|^{2}$

we obtain the following relationships between the structure functions and form factors for $v_{\mu}, \bar{v}_{\mu}$ QE scattering on free nucleons:

$\mathcal{W}_{1-\text { Qelastic }}^{\nu \text {-vector }}=\delta\left(v-\frac{Q^{2}}{2 M}\right) \tau\left|\mathcal{G}_{M}^{V}\left(Q^{2}\right)\right|^{2}$

$\mathcal{W}_{1 \text {-Qelastic }}^{\text {v-axial }}=\delta\left(v-\frac{Q^{2}}{2 M}\right)(1+\tau)\left|\mathcal{F}_{A}\left(Q^{2}\right)\right|^{2}$

$\mathcal{W}_{2 \text {-Qelastic }}^{\nu \text {-vector }}=\delta\left(v-\frac{Q^{2}}{2 M}\right)\left|\mathcal{F}_{V}\left(Q^{2}\right)\right|^{2}$

$\mathcal{W}_{2 \text {-Qelastic }}^{\nu \text {-axial }}=\delta\left(v-\frac{Q^{2}}{2 M}\right)\left|\mathcal{F}_{A}\left(Q^{2}\right)\right|^{2}$

$\mathcal{W}_{3-\text { Qelastic }}^{v}=\delta\left(v-\frac{Q^{2}}{2 M}\right)\left|2 \mathcal{G}_{M}^{V}\left(Q^{2}\right) \mathcal{F}_{A}\left(Q^{2}\right)\right|$

$\mathcal{W}_{4-\text { Qelastic }}^{\nu \text { vector }}=\delta\left(v-\frac{Q^{2}}{2 M}\right) \frac{1}{4}\left(\left|\mathcal{F}_{V}\left(Q^{2}\right)\right|^{2}-\left|\mathcal{G}_{M}^{V}\left(Q^{2}\right)\right|^{2}\right)$

$\mathcal{W}_{4-\text { Qelastic }}^{\text {vaxial }}$

$$
\begin{aligned}
= & \delta\left(v-\frac{Q^{2}}{2 M}\right) \frac{1}{4}\left[\mathcal{F}_{A}^{2}\left(Q^{2}\right)+\left(\frac{Q^{2}}{M^{2}}+4\right)\left|\mathcal{F}_{p}\left(Q^{2}\right)\right|^{2}\right] \\
& -\left(\mathcal{F}_{A}\left(Q^{2}\right)+2 \mathcal{F}_{P}\left(Q^{2}\right)\right)^{2}
\end{aligned}
$$

$\mathcal{W}_{5-\text { Qelastic }}^{\text {velor }}=\delta\left(v-\frac{Q^{2}}{2 M}\right) \frac{1}{2}\left|\mathcal{F}_{V}\left(Q^{2}\right)\right|^{2}$

$\mathcal{W}_{5 \text {-Qelastic }}^{\text {vaxial }}=\delta\left(v-\frac{Q^{2}}{2 M}\right) \frac{1}{2}\left|\mathcal{F}_{A}\left(Q^{2}\right)\right|^{2}$

The vector part of $\mathcal{W}_{4}$ and $\mathcal{W}_{5}$ are related to the vector part of $\mathcal{W}_{2}$ and $\mathcal{W}_{1}$ by the following expressions [48]:

$\mathcal{W}_{4}^{\text {vector }}=\mathcal{W}_{2}^{\text {vector }} \frac{M^{2} v^{2}}{Q^{4}}-\mathcal{W}_{1}^{\text {vector }} \frac{M^{2}}{Q^{2}}$

$\mathcal{W}_{5}^{\text {vector }}=\mathcal{W}_{2}^{\text {vector }} \frac{M \nu}{Q^{2}}$

Note that:

$\sigma_{T}^{\text {vector }} \propto \tau\left|\mathcal{G}_{M}^{V}\left(Q^{2}\right)\right|^{2} ; \quad \sigma_{T}^{\text {axial }} \propto(1+\tau)\left|\mathcal{F}_{A}\left(Q^{2}\right)\right|^{2}$ 
$\sigma_{L}^{\text {vector }} \propto\left(\mathcal{G}_{E}^{V}\left(Q^{2}\right)\right)^{2} ; \quad \sigma_{L}^{\text {axial }}=0$

Therefore, for QE $v_{\mu}$ and $\bar{v}_{\mu}$ scattering only $\mathcal{G}_{M}^{V}$ contributes to the vector part of the transverse virtual boson absorption cross section.

\section{References}

1. S. Fukuda et al., Phys. Rev. Lett. 85, 3999 (2000)

2. T. Toshito, hep-ex/0105023

3. D.G. Michael et al. (MINOS), Phys. Rev. Lett. 97, 191801 (2006)

4. P. Adamson et al. (MINOS), Phys. Rev. D 81, 072002 (2010)

5. P. Adamson et al. (MINOS Collaboration), First direct observation of muon antineutrino disappearance. FERMILAB-PUB-11-163PPD, BNL-94488-2010-JA. e-Print: arXiv:1104.0344 [hep-ex]

6. http://www-numi.fnal.gov/Minos/

7. A. Bodek, U.-k. Yang, Axial and vector structure functions for electron- and neutrino- nucleon scattering cross sections at all Q2 using effective leading order parton distribution functions. e-Print: arXiv:1011.6592 [hep-ph]

8. C. Andreopoulos (GENIE), Nucl. Instrum. Methods Phys. Res., Sect. A, Accel. Spectrom. Detect. Assoc. Equip. 614, 87 (2010)

9. H. Gallagher (NEUGEN), Nucl. Phys. Proc. Suppl. 112 (2002)

10. Y. Hayato (NEUT), Nucl. Phys. B, Proc. Suppl. 112, 171 (2002)

11. D. Casper (NUANCE), Nucl. Phys. B, Proc. Suppl. 112, 161 (2002). http://nuint.ps.uci.edu/nuance/

12. A. Bodek, J.L. Ritchie, Phys. Rev. D 23, 1070 (1981)

13. A.A. Aguilar-Arevalo et al. (MiniBooNE), Phys. Rev. Lett. 98, 231801 (2007)

14. A.A. Aguilar-Arevalo et al., Measurement of the neutrino component of an anti-neutrino beam observed by a non-magnetized detector. e-Print: arXiv:1102.1964 [hep-ex]

15. C. Juszczak, J.T. Sobczyk, J. Zmuda, Phys. Rev. C 82, 045502 (2010)

16. A.A. Aguilar-Arevalo (MiniBooNE Collaboration), Phys. Rev. D 81, 092005 (2010). http://www-boone.fnal.gov/for_physicists/ data_release/ccqe

17. Y. Itow et al. (T2K). arXiv:hep-ex/0106019

18. M.H. Ahn et al. (K2K), Phys. Rev. D 74, 072003 (2006). http://neutrino.kek.jp/

19. A. Bodek, S. Avvakumov, R. Bradford, H. Budd, Eur. Phys. J. C 53, 349 (2008)

20. A.V. Butkevich, Phys. Rev. C 82, 055501 (2010) and references therein
21. J.J. Kelly, Phys. Rev. C 70, 068202 (2004)

22. S. Galster et al., Nucl. Phys. B 32, 221 (1971)

23. P.E. Bosted, Phys. Rev. C 51, 409 (1995)

24. B. Bartoli et al., Riv. Nuovo Cimento 2, 241 (1972)

25. R.F. Wegenbrunn et al., Few-Body Syst., Suppl. 14, 411 (2003). hep-ph/0212190

26. M. Sajjad Athar, S. Ahmad, S.K. Singh, Phys. Rev. D 75, 093003 (2007)

27. T. Leitner, L. Alvarez-Ruso, U. Mosel, Phys. Rev. C 73, 065502 (2006)

28. K. Tsushima, H. Kim, K. Saito, Phys. Rev. C 70, 038501 (2004)

29. K.S. Kuzmin, V.V. Lyubushkin, V.A. Naumov, Eur. Phys. J. C 54, 517-538 (2008). arXiv:0712.4384 [hep-ph]

30. V. Lyubushkin et al. (NOMAD Collaboration), Eur. Phys. J. C 63, 355 (2009)

31. Q. Wu et al. (NOMAD Collaboration), Phys. Lett. B 60, 19 (2008)

32. F.M. Steffens, K. Tsushima, Phys. Rev. D 70, 094040 (2004)

33. T.W. Donnelly, I. Sick, Phys. Rev. C 60, 065502 (1999)

34. C. Maieron, J.E. Amaro, M.B. Barbaro, J.A. Caballero, T.W. Donnelly, C.F. Williamson, Phys. Rev. C 80, 035504 (2009)

35. J.E. Amaro, M.B. Barbaro, J.A. Caballero, T.W. Donnelly, C.F. Williamson, Phys. Lett. B 696, 151 (2011)

36. J. Carlson, J. Jourdan, R. Schiavilla, I. Sick, Phys. Rev. C 65, 024002 (2002)

37. M. Martini, M. Ericson, G. Chanfray, J. Marteau, Phys. Rev. C 80, 065501 (2009)

38. M. Martini, M. Ericson, G. Chanfray, J. Marteau, Phys. Rev. C 81, 045502 (2010)

39. A. Bodek, C. Keppel, M.E. Christy (JUPITER Collaboration), Jefferson Lab experiment E04-001

40. C. Maieron, T.W. Donnelly, I. Sick, Phys. Rev. C 65, 025502 (2002)

41. M.E. Christy, P.E. Bosted, Phys. Rev. C 81, 055213 (2010)

42. P.E. Bosted, M.E. Christy, Phys. Rev. C 77, 065206 (2008)

43. V. Mamyan, Ph.D. dissertation. University of Virginia (2010)

44. O. Lalakulich, W. Melnitchouk, E.A. Paschos, Phys. Rev. C 75, 015202 (2007)

45. S.J. Yu, M.H. Reno, Phys. Rev. D 82, 033010 (2010)

46. S. Kretzer, M.H. Reno, Phys. Rev. D 66, 113007 (2002)

47. C.H. Albright, C. Jarlskog, Nucl. Phys. B 84, 467 (1975)

48. O. Lalakulich, E.A. Paschos, Phys. Rev. D 71, 074003 (2005)

49. C.H. Llewellyn Smith, Phys. Rep. 3C (1972)

50. E.A. Paschos, Electroweak Theory (Cambridge University Press, Cambridge, 2007)

51. http://minerva.fnal.gov/ 